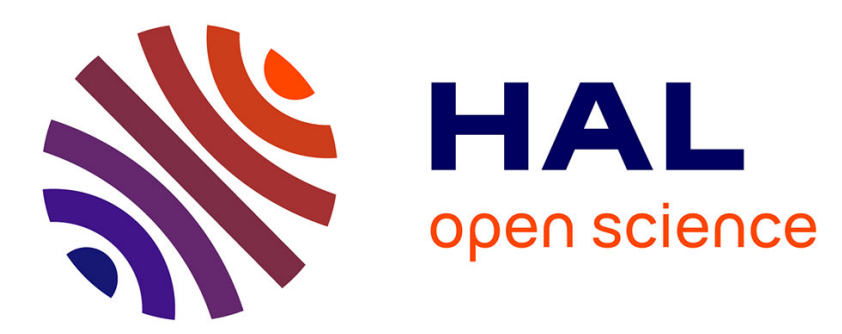

\title{
A diffuse interface Lox/hydrogen transcritical flame model
}

\author{
Pierre Gaillard, Vincent Giovangigli, Lionel Matuszewski
}

\section{To cite this version:}

Pierre Gaillard, Vincent Giovangigli, Lionel Matuszewski. A diffuse interface Lox/hydrogen transcritical flame model. Combustion Theory and Modelling, 2016, 10.1080/13647830.2016.1150518 . hal-01308378

\section{HAL Id: hal-01308378 https://hal.science/hal-01308378}

Submitted on 29 Apr 2016

HAL is a multi-disciplinary open access archive for the deposit and dissemination of scientific research documents, whether they are published or not. The documents may come from teaching and research institutions in France or abroad, or from public or private research centers.
L'archive ouverte pluridisciplinaire HAL, est destinée au dépôt et à la diffusion de documents scientifiques de niveau recherche, publiés ou non, émanant des établissements d'enseignement et de recherche français ou étrangers, des laboratoires publics ou privés. 


\section{A diffuse interface Lox/hydrogen transcritical flame model}

\section{Pierre Gaillard, Vincent Giovangigli \& Lionel Matuszewski}

To cite this article: Pierre Gaillard, Vincent Giovangigli \& Lionel Matuszewski (2016): A diffuse interface Lox/hydrogen transcritical flame model, Combustion Theory and Modelling, DOI: 10.1080/13647830.2016.1150518

To link to this article: http://dx.doi.org/10.1080/13647830.2016.1150518

曲 Published online: 30 Mar 2016.

Submit your article to this journal $[\pi$

山 Article views: 10

Q View related articles ¿

View Crossmark data ¿ 


\title{
A diffuse interface Lox/hydrogen transcritical flame model
}

\author{
Pierre Gaillard $^{\mathrm{a}}$, Vincent Giovangigli ${ }^{\mathrm{a}, \mathrm{b} *}$ and Lionel Matuszewski ${ }^{\mathrm{a}}$ \\ ${ }^{a}$ ONERA DEFA, Palaiseau, France; ${ }^{b}$ CMAP-CNRS, Ecole polytechnique, Palaiseau, France
}

(Received 20 June 2015; accepted 26 January 2016)

\begin{abstract}
We present a diffuse-interface all-pressure flame model that transitions smoothly between subcritical and supercritical conditions. The model involves a non-equilibrium liquid/gas diffuse interface of van der Waals/Korteweg type embedded into a non-ideal multicomponent reactive fluid. The multicomponent transport fluxes are evaluated in their thermodynamic form in order to avoid singularities at thermodynamic mechanical stability limits. The model also takes into account condensing liquid water in order to avoid thermodynamic chemical instabilities. The resulting equations are used to investigate the interface between cold dense and hot light oxygen as well as the structure of diffusion flames between cold dense oxygen and gaseous-like hydrogen at all pressures, either subcritical or supercritical.
\end{abstract}

Keywords: supercritical; transcritical; diffuse interface; flame

\section{Introduction}

The injection of reactants in cryogenic combustion engines is often performed at supercritical pressure - in order to increase engine efficiency - and below the critical temperature for storage purposes. Experimental campaigns on cryotechnic benches such as MASCOTTE have been devoted to combustion in such extreme conditions [1,2]. Numerical simulations have also been concerned with supercritical flames in laminar [3-10] as well as turbulent [11-13] flows with non-ideal thermodynamics predicted with cubic equations of states. Numerical simulations of supercritical laminar flames have notably been performed by El Gamal et al. [5] and Giovangigli et al. [10] for plane flames and by Okongo and Bellan [6] for mixing layers, whereas counterflow flames or droplets have notably been investigated by Saur et al. [3], Daou et al. [4], Ribert et al. [7], Pons et al. [8], and Giovangigli and Matuszewski [10]. Such simulations may be transcritical with respect to temperature but are supercritical with respect to pressure and did not involve or require diffuse interfaces.

Diffuse interface models describe the continuous change of fluid properties across liquid/gas interfaces in subcritical conditions. Such diffuse interface models may be derived from the second gradient theory of Van der Waals, Korteweg, Cahn and Hilliard [14-26]. Another type of diffuse interface model, which lies out of the scope of the present work, is that of multifluid models typically obtained through averaging processes [27-34]. The thermodynamics of second gradient diffuse interface models has been built by van der Waals $[14,15]$ using a gradient squared term in the free energy and the corresponding capillary tensor has been derived by Korteweg [16]. Second gradient theory has been further extended to binary fluids by Cahn and Hilliard [17,18]. Statistical mechanics of systems 
that are highly inhomogeneous has also led to mean field theories of liquid/gas interfaces and to direct links between capillary phenomena and intermolecular forces as established by Rowlinson and Widom [19]. Second gradient diffuse interface models have been successfully used to describe near critical points [19], the dynamics of contact lines [20], as well as complex liquid/gas interfaces with topological changes [21-23], and are compatible with the limiting free boundary problem when the interface thickness goes to zero [21]. In the high pressure domain, Dahms and Oefelein have used diffuse interface models to investigate locally subcritical vaporising fronts using the linear gradient theory $[25,26]$. The linear gradient theory is a simplified formulation that allows isothermal vaporising fronts to be investigated for given density boundary conditions assuming linear dependencies among species densities and avoiding having to solve boundary value problems. These studies have led to a comprehensive analysis of the complex physics of vaporising interfaces at high pressure $[25,26]$.

During ignition in rocket engines, however, a transition may occur from subcritical to supercritical pressure conditions and the resulting dynamics cannot be described by current available flame models. Similarly, in diesel engines, the cylinder pressure may temporarily exceeds the thermodynamic critical pressure of the injected fuel and major differences may occur in the jet dynamics. There is thus a need for flame models that transition smoothly from subcritical to supercritical pressure conditions. In this paper, we introduce such a diffuse interface transcritical flame model using several ingredients.

The domain of applicability of diffuse interface models is first extended to the supercritical domain, which seems new to the best of the authors' knowledge. The non-equilibrium diffuse interface model is then embedded into a non-ideal multicomponent reactive fluid framework. The multicomponent fluxes are notably evaluated in their thermodynamic form in order to avoid singularities at thermodynamic mechanical stability limits. Binary phase diagrams of oxygen and water are investigated and liquid water is taken into account in order to avoid thermodynamic chemical instabilities. The resulting boundary value problem is solved for strained vaporising oxygen interfaces as well as oxygen/hydrogen diffusion flames at all pressure, either subcritical or supercritial.

The equations governing diffuse interfaces are presented in Section 2. The small Mach limit for flat interfaces, self-similar equations, bulk phases thermodynamics and non-ideal transport fluxes are addressed in Section 3. Numerical simulations of oxygen interfaces are then presented in Section 4 and numerical simulations of oxygen/hydrogen flames are presented in Section 5.

\section{Korteweg fluids}

We present the van der Waals free energy function and thermodynamics for multicomponent mixtures and the corresponding Korteweg fluid equations. The free energy contains extra gradient squared terms typically associated with long range molecular interactions [14-21].

\subsection{Van der Waals free energy}

The free energy per unit volume in second gradient theory is typically of the form

$$
\mathcal{F}=\mathcal{F}^{0}+\sum_{i, j \in \mathfrak{S}} \kappa_{i j} \nabla \rho_{i} \cdot \nabla \rho_{j}
$$


where $\mathfrak{S}=\{1, \ldots, n\}$ denotes the species indexing set, $n$ the number of species, $\mathcal{F}^{0}$ the bulk free energy, $\rho_{i}$ the partial density of the $i$ th species, $\nabla$ the usual differential operator and $\kappa_{i j}, i, j \in \mathfrak{S}$, the capillary coefficients. The superscript ${ }^{0}$ is used to denote standard bulk phase thermodynamic properties that do not involve gradients. The free energy $\mathcal{F}^{0}$ only depends on the partial densities $\rho_{1}, \ldots, \rho_{n}$ and the absolute temperature $T$ whereas the gradient squared term $\sum_{i, j \in \mathfrak{S}} \kappa_{i j} \nabla \rho_{i} \cdot \nabla \rho_{j}$ in $\mathcal{F}$ represents the excess free energy of the interfacial region. This gradient term may be interpreted from attractive long range molecular interactions and the capillary coefficients $\kappa_{i j}$ related to the pair correlation function [19].

From the expression of the free energy (1) and the classical thermodynamic relation $\mathrm{d} \mathcal{F}^{0}=-\mathcal{S}^{0} \mathrm{~d} T+\sum_{i \in \mathfrak{S}} g_{i}^{0} \mathrm{~d} \rho_{i}$, assuming that the capillarity coefficients $\kappa_{i j}, i, j \in \mathfrak{S}$, are constant for the sake of simplicity, we obtain that

$$
\mathrm{d} \mathcal{F}=-\mathcal{S}^{0} \mathrm{~d} T+\sum_{i \in \mathfrak{S}} g_{i}^{0} \mathrm{~d} \rho_{i}+\sum_{i \in \mathfrak{S}} \boldsymbol{\phi}_{i} \cdot \mathrm{d} \nabla \rho_{i},
$$

where $\mathcal{S}^{0}$ denotes the bulk entropy per unit volume, $g_{i}^{0}$ the bulk Gibbs function of the $i$ th species per unit mass, and the vectors $\phi_{i}, i \in \mathfrak{S}$, are given by

$$
\boldsymbol{\phi}_{i}=\sum_{j \in \mathfrak{S}}\left(\kappa_{i j}+\kappa_{j i}\right) \nabla \rho_{j}, \quad i \in \mathfrak{S} .
$$

Using the thermodynamic relations $\partial_{T} \mathcal{F}=-\mathcal{S}$ and $\partial_{\rho_{i}} \mathcal{F}=g_{i}, i \in \mathfrak{S}$, where $\mathcal{S}$ denotes the entropy per unit volume and $g_{i}$ the Gibbs function per unit mass of the $i$ th species, the identity (2) implies that $\mathcal{S}=\mathcal{S}^{0}$ and $g_{i}=g_{i}^{0}$.

The other thermodynamic functions are easily obtained by using the expression (1) for the free energy $\mathcal{F}$, the identity $\mathcal{S}=\mathcal{S}^{0}$, and standard thermodynamic relations. The Gibbs function $\mathcal{G}$ and the enthalpy per unit volume $\mathcal{H}$ are found to be given by their standard values $\mathcal{G}=\mathcal{G}^{0}$ and $\mathcal{H}=\mathcal{H}^{0}$, whereas the energy per unit volume $\mathcal{E}$ and the pressure $p$ read

$$
\mathcal{E}=\mathcal{E}^{0}+\sum_{i, j \in \mathfrak{S}} \kappa_{i j} \nabla \rho_{i} \cdot \nabla \rho_{j}, \quad p=p^{0}-\sum_{i, j \in \mathfrak{S}} \kappa_{i j} \nabla \rho_{i} \cdot \nabla \rho_{j}
$$

Denoting respectively by $e, s, f, g$ and $h$ the energy, entropy, free energy, Gibbs function and enthalpy per unit mass, by $\rho=\sum_{i \in \mathfrak{S}} \rho_{i}$ the mass density, and $\mathrm{y}_{i}$ the mass fraction of the $i$ th species, we naturally have $\mathcal{E}=\rho e, \mathcal{S}=\rho s, \mathcal{F}=\rho f, \mathcal{G}=\rho g, \mathcal{H}=\rho h, \rho_{i}=\rho \mathrm{y}_{i}$, $i \in \mathfrak{S}$, and the Gibbs relation for the entropy $s$ is of the form

$$
T \mathrm{~d} s=\mathrm{d} e-\frac{p}{\rho^{2}} \mathrm{~d} \rho-\sum_{i \in \mathfrak{S}} g_{i}^{0} \mathrm{~d} \mathbf{y}_{i}-\sum_{i \in \mathfrak{S}} \frac{1}{\rho} \boldsymbol{\phi}_{i} \cdot \mathrm{d} \nabla \rho_{i},
$$

where $d$ denotes the differentiation operator. We will deduce from this relation (5) an entropy balance equation and next obtain the structure of the mass, momentum and energy fluxes.

Physical values for the capillary coefficients $\kappa_{i j}, i, j \in \mathfrak{S}$, are generally temperature dependent and may be obtained by fitting surface tension experimental data [24]. Such coefficients are typically of the form of an exponential of a polynomial in $\log \left(1-T / T^{\mathrm{c}}\right)$, 
where $T^{\mathrm{c}}$ is a critical temperature and are thus only defined in the subcritical domain [24]. The resulting diffuse interfaces are then very thin, typically of the order of a few nanometers $[19,25,26]$. The diffuse interface model allows the structure of such interfaces to be faithfully reproduced using numerical grids with nanometer characteristic lengths $[25,26]$.

Although such detailed simulations can be obtained with the diffuse interface model, we want to obtain in this paper computationally feasible numerical flame models taking into account the limitation on grid resolution. In this situation, one is forced to use larger values of the capillary coefficients leading to artificially thickened interfaces and to larger values of surface tension. This implicitly assumes that there is a scale separation so that interface thickening and the corresponding larger numerical surface tension do not significantly modify the flame under investigation.

In addition, our aim is to extend the diffuse interface model to supercritical conditions in order to encompass all pressures. We thus have to extend the domain of definition of capillary coefficients with finite values for all pressures and, in practice, we have used constant values for capillary parameters. In the subcritical domain, the resulting diffuse interface model then allows the computation of a continuous liquid/gas interface, whereas in the supercritical domain the model may ease the numerical solution of sharp pseudovaporising transition layers. Nonequilibrium interfaces are considered in this work and the link with equilibrium interfaces is addressed in Appendix A.

\subsection{Governing equations}

The total mass conservation equation may be written

$$
\partial_{t} \rho+\nabla \cdot(\rho \boldsymbol{v})=0
$$

where $v$ denotes the fluid velocity, and the species mass conservation equations are of the form

$$
\partial_{t} \rho_{i}+\nabla \cdot\left(\rho_{i} \boldsymbol{v}\right)+\nabla \cdot \mathcal{J}_{i}=m_{i} \omega_{i}, \quad i \in \mathfrak{S},
$$

where $\mathcal{J}_{i}$ is the mass flux of the $i$ th species, $m_{i}$ the molar mass of the $i$ th species, and $\omega_{i}$ the molar rate of production of the $i$ th species. The chemical source terms $\omega_{i}, i \in \mathfrak{S}$, and the diffusive fluxes $\mathcal{J}_{i}, i \in \mathfrak{S}$, satisfy the mass conservation constraints

$$
\sum_{i \in \mathfrak{S}} \mathcal{J}_{i}=0, \quad \sum_{i \in \mathfrak{S}} m_{i} \omega_{i}=0
$$

so that by summing the species conservation equations (7) we recover the total mass conservation equation (6). The momentum conservation equation reads

$$
\partial_{t}(\rho \boldsymbol{v})+\nabla \cdot(\rho \boldsymbol{v} \otimes \boldsymbol{v})+\nabla \cdot \mathcal{P}=0
$$

where $\mathcal{P}$ is the symmetric pressure tensor and the total energy conservation equation may be written

$$
\partial_{t}\left(\rho\left(e+\frac{1}{2}|\boldsymbol{v}|^{2}\right)\right)+\nabla \cdot\left(\rho \boldsymbol{v}\left(e+\frac{1}{2}|\boldsymbol{v}|^{2}\right)\right)+\nabla \cdot(\mathcal{Q}+\mathcal{P} \cdot \boldsymbol{v})=0
$$


where $\mathcal{Q}$ denotes the total heat flux. Multiplying the momentum conservation equation (9) by the velocity vector $\boldsymbol{v}$ and subtracting the result from the total energy conservation equation (10) yields a conservation equation for the internal energy as in standard fluids.

\subsection{Transport fluxes}

The transport fluxes are derived by expressing the entropy production rate in terms of the fluxes and the variable gradients and proceeding as in the thermodynamics of irreversible processes $[21,23,35]$. By using Gibbs relation (5), it is obtained after some algebra that

$$
\begin{aligned}
\rho\left(\partial_{t} s+\boldsymbol{v} \cdot \nabla s\right)+ & \nabla \cdot\left(\frac{\mathcal{Q}}{T}-\sum_{i \in \mathfrak{S}} \frac{\tilde{g}_{i} \mathcal{J}_{i}}{T}+\sum_{i \in \mathfrak{S}} \frac{\boldsymbol{\phi}_{i}}{T}\left(m_{i} \omega_{i}-\nabla \cdot \mathcal{J}_{i}-\rho_{i} \nabla \cdot \boldsymbol{v}\right)\right) \\
= & -\frac{1}{T}\left(\mathcal{P}-p \boldsymbol{I}-\sum_{i \in \mathfrak{S}}\left(\nabla \rho_{i} \otimes \boldsymbol{\phi}_{i}-\rho_{i} \nabla \cdot \boldsymbol{\phi}_{i} \boldsymbol{I}\right)\right): \nabla \boldsymbol{v} \\
& -\left(\mathcal{Q}+\sum_{i \in \mathfrak{S}} \boldsymbol{\phi}_{i}\left(m_{i} \omega_{i}-\nabla \cdot \mathcal{J}_{i}-\rho_{i} \nabla \cdot \boldsymbol{v}\right)\right) \cdot \frac{\nabla T}{T^{2}} \\
& -\sum_{i \in \mathfrak{S}} \mathcal{J}_{i} \cdot \nabla\left(\frac{\widetilde{g}_{i}}{T}\right)-\sum_{i \in \mathfrak{S}} \frac{\widetilde{g}_{i} m_{i} \omega_{i}}{T},
\end{aligned}
$$

where $\tilde{g}_{i}=g_{i}^{0}-\nabla \cdot \boldsymbol{\phi}_{i}$ denotes the generalised Gibbs free energy of the $i$ th species. Proceeding as in the thermodynamics of irreversible processes, and using the Curie principle, we first deduce from the expression of entropy production (11), that the pressure tensor and the total heat flux are of the form

$$
\begin{aligned}
& \mathcal{P}=p \boldsymbol{I}+\sum_{i \in \mathfrak{S}}\left(\nabla \rho_{i} \otimes \boldsymbol{\phi}_{i}-\rho_{i} \nabla \cdot \boldsymbol{\phi}_{i} \boldsymbol{I}\right)+\boldsymbol{\Pi}, \\
& \mathcal{Q}=-\sum_{i \in \mathfrak{S}} \boldsymbol{\phi}_{i}\left(m_{i} \omega_{i}-\nabla \cdot \mathcal{J}_{i}-\rho_{i} \boldsymbol{\nabla} \cdot \boldsymbol{v}\right)+\boldsymbol{q},
\end{aligned}
$$

where $\boldsymbol{\Pi}$ is the viscous tensor and $\boldsymbol{q}$ the dissipative heat flux, and, on the other hand, that the dissipative transport fluxes $\Pi, \mathcal{J}_{i}, i \in \mathfrak{S}$, and $\boldsymbol{q}$ are of the form

$$
\begin{gathered}
\boldsymbol{\Pi}=-\mathfrak{v} \nabla \cdot \boldsymbol{v} \boldsymbol{I}-\eta\left(\nabla \boldsymbol{v}+\nabla \boldsymbol{v}^{t}-\frac{2}{3} \nabla \cdot \boldsymbol{v} \boldsymbol{I}\right), \\
\mathcal{J}_{i}=-\sum_{j \in \mathfrak{S}} L_{i j} \nabla\left(\frac{\widetilde{g}_{j}}{T}\right)-L_{i e} \nabla\left(\frac{-1}{T}\right), \\
\boldsymbol{q}=-\sum_{i \in \mathfrak{S}} L_{e i} \nabla\left(\frac{\widetilde{g}_{i}}{T}\right)-L_{e e} \nabla\left(\frac{-1}{T}\right),
\end{gathered}
$$

where $\mathfrak{v}$ denotes the volume viscosity, $\eta$ the shear viscosity and $L_{i j}, i, j \in \mathfrak{S} \cup\{e\}$, the mass and heat transport coefficients. The matrix of mass and heat transport coefficients $L$ defined by $L=\left(L_{i j}\right)_{i, j \in \mathfrak{S} \cup\{e\}}$ is symmetric positive semi-definite with nullspace spanned by the vector $(1, \ldots, 1,0)^{t}$ as for ordinary fluids [35]. The fluxes $\mathcal{J}_{i}, i \in \mathfrak{S}$, and $\boldsymbol{q}$ are obtained here in their thermodynamic form, which will be useful in the following. In the pressure tensor 
$\mathcal{P}$, the component $\mathcal{P}-p^{0} \boldsymbol{I}-\Pi$ represents the reversible part associated with interstitial work.

\subsection{A simplified framework}

We introduce in this section a simplified model obtained by assuming that all capillary coefficients are equal. This model is well justified when there are large density gradients at the interface and when the interface composition is essentially frozen. We therefore assume for the purpose of simplicity that

$$
\kappa_{i j}=\frac{1}{2} \kappa, \quad i, j \in \mathfrak{S}
$$

where $\kappa$ is a constant and species independent capillary coefficient. We then obtain from (1), (3) and the definition of the generalised Gibbs free energies $\widetilde{g}_{i}, i \in \mathfrak{S}$, that

$$
\begin{gathered}
\mathcal{F}=\mathcal{F}^{0}+\frac{1}{2} \kappa|\nabla \rho|^{2}, \\
\boldsymbol{\phi}_{i}=\kappa \nabla \rho, \quad i \in \mathfrak{S}, \\
\widetilde{g}_{i}=g_{i}^{0}-\kappa \Delta \rho, \quad i \in \mathfrak{S},
\end{gathered}
$$

where $\Delta \rho$ denotes the Laplacian of the density.

Using the general relations expressing the transport fluxes (12)-(16), the simplified expressions (19) and (20), the mass constraints $\sum_{i \in \mathfrak{S}} m_{i} \omega_{i}=0$ and $\sum_{i \in \mathfrak{S}} \mathcal{J}_{i}=0$, as well as $\sum_{i \in \mathfrak{S}} L_{i j}=\sum_{j \in \mathfrak{S}} L_{i j}=0$ and $\sum_{j \in \mathfrak{S}} L_{e j}=\sum_{i \in \mathfrak{S}} L_{i e}=0$, we obtain after some algebra that (12) and (13) simplify to

$$
\begin{gathered}
\mathcal{P}=p \boldsymbol{I}+\kappa \nabla \rho \otimes \nabla \rho-\kappa \rho \Delta \rho \boldsymbol{I}+\boldsymbol{\Pi}, \\
\mathcal{Q}=\kappa \rho \nabla \cdot \boldsymbol{v} \nabla \rho+\boldsymbol{q},
\end{gathered}
$$

and moreover that (15) and (16) simplify to

$$
\begin{gathered}
\mathcal{J}_{i}=-\sum_{j \in \mathfrak{S}} L_{i j} \nabla\left(\frac{g_{j}^{0}}{T}\right)-L_{i e} \nabla\left(\frac{-1}{T}\right), \quad i \in \mathfrak{S}, \\
\boldsymbol{q}=-\sum_{i \in \mathfrak{S}} L_{e i} \nabla\left(\frac{g_{i}^{0}}{T}\right)-L_{e e} \nabla\left(\frac{-1}{T}\right) .
\end{gathered}
$$

The dissipative fluxes $\mathcal{J}_{i}, i \in \mathfrak{S}$, and $\boldsymbol{q}$ are in their thermodynamic form written directly in terms of the complete gradients of the bulk chemical potentials $g_{j}^{0} / T, j \in \mathfrak{S}$, and of the usual thermodynamic thermal variable $-1 / T$. In the pressure tensor $\mathcal{P}$ the new contributions are $-\kappa \rho \Delta \rho \boldsymbol{I}$, the Korteweg tensor $\kappa \nabla \rho \otimes \nabla \rho$ and the pressure $p$ also differs from $p^{0}$. These extra terms associated with capillary phenomena do not produce entropy and are associated with interstitial work which is a reversible process, at variance with diffusion, chemistry and thermal conductivity. The diffusive fluxes $\mathcal{J}_{i}, i \in \mathfrak{S}$, correspond to their standard 
expression but the heat flux $\mathcal{Q}$ also contains a supplementary term $\kappa \rho \nabla \cdot v \nabla \rho$ induced by capillarity.

We also note that by using the simplified expressions $\widetilde{g}_{i}=g_{i}^{0}-\kappa \Delta \rho, i \in \mathfrak{S}$, and the mass constraint $\sum_{i \in \mathfrak{S}} m_{i} \omega_{i}=0$, the entropy production due to chemistry simplifies to the form $-\sum_{i \in \mathfrak{S}} \widetilde{g}_{i} m_{i} \omega_{i} / T=-\sum_{i \in \mathfrak{S}} g_{i}^{0} m_{i} \omega_{i} / T$ only involving the bulk thermodynamics chemical potentials $g_{i}^{0} / T, i \in \mathfrak{S}$.

\subsection{Production rates}

We consider an arbitrary complex chemical reaction mechanism of the form

$$
\sum_{i \in \mathfrak{S}} v_{i j}^{\mathrm{f}} \mathfrak{M}_{i} \rightleftarrows \sum_{i \in \mathfrak{S}} v_{i j}^{\mathrm{b}} \mathfrak{M}_{i}, \quad j \in \mathfrak{R}
$$

where $v_{i j}^{\mathrm{f}}$ and $v_{i j}^{\mathrm{b}}$ are the forward and reverse stoichiometric coefficients of the $i$ th species in the $j$ th reaction, $\mathfrak{M}_{i}$ the symbol of the $i$ th molecule, $\mathfrak{R}=\left\{1, \ldots, n^{r}\right\}$ the indexing set of chemical reactions and $n^{r}$ the number of chemical reactions. The production rates are given by the usual expression

$$
\omega_{i}=\sum_{j \in \Re} v_{i j} \tau_{j}
$$

where $v_{i j}=v_{i j}^{\mathrm{b}}-v_{i j}^{\mathrm{f}}$ is the overall stoichiometric coefficient of the $k$ th species in the $j$ th reaction and $\tau_{j}$ is the rate of progress of the $j$ th reaction. The following vectors are introduced for convenience:

$$
v_{j}=\left(\begin{array}{c}
v_{1 j} \\
\vdots \\
v_{n j}
\end{array}\right), \quad v_{j}^{\mathrm{f}}=\left(\begin{array}{c}
v_{1 j}^{\mathrm{f}} \\
\vdots \\
v_{n j}^{\mathrm{f}}
\end{array}\right), \quad v_{j}^{\mathrm{b}}=\left(\begin{array}{c}
v_{1 j}^{\mathrm{b}} \\
\vdots \\
v_{n j}^{\mathrm{b}}
\end{array}\right), \quad \mu=\left(\begin{array}{c}
\mu_{1} \\
\vdots \\
\mu_{n}
\end{array}\right) \text {, }
$$

where $\mu_{i}=m_{i} g_{i}^{0} / R T$ is the reduced bulk chemical potential. The reaction rates of progress given by statistical thermodynamics $[9,36,37]$ are of the form

$$
\tau_{j}=\mathcal{K}_{j}\left(\exp \left\langle v_{j}^{\mathrm{f}}, \mu\right\rangle-\exp \left\langle v_{j}^{\mathrm{b}}, \mu\right\rangle\right), \quad j \in \mathfrak{R}
$$

where $\mathcal{K}_{j}$ is the symmetric constant of the $j$ th reaction. The resulting expression of entropy production in (11) is finally given by

$$
\begin{aligned}
\sum_{i, j \in \mathfrak{S} \cup\{e\}} L_{i j} & \nabla\left(\frac{g_{i}^{0}}{T}\right) \cdot \nabla\left(\frac{g_{j}^{0}}{T}\right)+\frac{\mathfrak{v}}{T}(\nabla \cdot v)^{2}+\frac{\eta}{2 T}|\mathrm{~S}|^{2} \\
& +\sum_{j \in \mathfrak{R}} R \mathcal{K}_{j}\left(\left\langle v_{j}^{\mathrm{f}}, \mu\right\rangle-\left\langle v_{j}^{\mathrm{b}}, \mu\right\rangle\right)\left(\exp \left\langle v_{j}^{\mathrm{f}}, \mu\right\rangle-\exp \left\langle v_{j}^{\mathrm{b}}, \mu\right\rangle\right),
\end{aligned}
$$

where we have let $g_{e}^{0}=-1$ for convenience, where $S$ denotes the deviatoric part of the strain rate tensor $\mathbf{S}=\boldsymbol{\nabla} \boldsymbol{v}+\boldsymbol{\nabla} \boldsymbol{v}^{t}-\frac{2}{3} \boldsymbol{\nabla} \cdot \boldsymbol{v} \boldsymbol{I}$, and $|A|^{2}$ the Frobenius norm $|A|^{2}=A: A=\sum_{i j} a_{i j}^{2}$ of a tensor $A=\left(a_{i j}\right)$, so that entropy production appears as a sum of non-negative terms. 
Remark 1: Using the generalised Gibbs function $\tilde{g}_{i}=g_{i}^{0}-\kappa \Delta \rho, i \in \mathfrak{S}$, in (26) would not change the expression for the rates of progress. Considering the $j$ th reaction and letting $\tilde{\mu}_{i}=m_{i} \widetilde{g}_{i} / R T$ and $\tilde{\mu}=\left(\tilde{\mu}_{1}, \ldots, \tilde{\mu}_{n}\right)^{t}$ we indeed obtain $\left\langle v_{j}^{\mathrm{f}}, \tilde{\mu}\right\rangle=\left\langle v_{j}^{\mathrm{f}}, \mu\right\rangle-\sigma_{j} \kappa \Delta \rho$ and $\left\langle v_{j}^{\mathrm{b}}, \tilde{\mu}\right\rangle=\left\langle v_{j}^{\mathrm{b}}, \mu\right\rangle-\sigma_{j} \kappa \Delta \rho$, where $\sigma_{j} R T=\sum_{i \in \mathfrak{S}} v_{i j}^{\mathrm{f}} m_{i}=\sum_{i \in \mathfrak{S}} v_{i j}^{\mathrm{b}} m_{i}$, the last equality resulting from mass conservation in the $j$ th reaction. The extra factors $\exp \left(-\sigma_{j} \kappa \Delta \rho\right)$ obtained by using the generalised Gibbs functions $\widetilde{g}_{i}, i \in \mathfrak{S}$, instead of $g_{i}^{0}, i \in \mathfrak{S}$, may thus be absorbed in the symmetric reaction constant $\mathcal{K}_{j}$ of the $j$ th chemical reaction. In the vaporising zone of dense oxygen, where gradients of density are high, chemistry effects are anyway limited.

\section{Non-ideal strained diffuse interfaces}

We complete the Korteweg fluid model presented in Section 2 by investigating the small Mach number limit for flat interfaces as well as the equations governing strained self-similar flows. We address the non-ideal thermodynamics of the bulk phases associated with the Soave-Redlich-Kwong (SRK) equation of state. We finally discuss the thermodynamic form of multicomponent fluxes and the evaluation of the transport matrix $L$ at mechanical thermodynamic instabilities.

\subsection{Small Mach number limit for flat interfaces}

In a supercritical mixing layer or in a typical diffusion laminar flame, the local Mach number is generally small. It is therefore relevant to investigate the small Mach number limit of the diffuse interface model presented in the previous sections which is especially convenient for flat interfaces. Proceeding as for classical fluids [38], we expand the bulk thermodynamic pressure $p^{0}$ in powers of the square of the Mach number $\epsilon$

$$
p^{0}=\bar{p}^{0}+\epsilon^{2} \widetilde{p}^{0}
$$

with $\bar{p}^{0}$ denoting the zeroth order bulk pressure and $\widetilde{p}^{0}$ the fluid dynamic perturbation. Bulk thermodynamic properties may thus be evaluated at the zeroth order pressure $\bar{p}^{0}$. The unknowns other than pressure are also expanded in terms of the square of the Mach number and are denoted as their zeroth value in order to simplify notation. The thermodynamic pressure $p=p^{0}-\frac{1}{2} \kappa|\nabla \rho|^{2}$ can then be written $p=\bar{p}^{0}-\frac{1}{2} \kappa|\nabla \rho|^{2}+\epsilon^{2} \tilde{p}$ and, substituting this expansion in the momentum equation, it is found at zeroth order that

$$
\nabla \cdot\left(\bar{p}^{0} \boldsymbol{I}-\frac{1}{2} \kappa|\nabla \rho|^{2} \boldsymbol{I}+\kappa \nabla \rho \otimes \nabla \rho-\kappa \rho \Delta \rho \boldsymbol{I}\right)=0 .
$$

In the absence of capillary effects, when $\kappa=0$ we recover the usual relation $\nabla \cdot\left(\bar{p}^{0} \boldsymbol{I}\right)=$ $\nabla \bar{p}^{0}=0$ which yields that the zeroth order bulk thermodynamic pressure $\bar{p}^{0}$ is spatially uniform. The situation is more complex with capillary effects so that the small Mach number limit (28) is not very practical without further assumptions.

In order to simplify the model, we assume a planar interface with all quantities only depending spatially on a normal coordinate $\zeta$ so that $\rho=\rho(t, \zeta), \bar{p}^{0}=\bar{p}^{0}(t, \zeta), T=T(t$, $\zeta)$, and $\boldsymbol{\phi}=\boldsymbol{\phi}(t, \zeta)$. In this framework, the zeroth order pressure tensor $\overline{\mathcal{P}}$ is of the form $\overline{\mathcal{P}}=\left(\bar{p}^{0}-\frac{1}{2} \kappa \rho^{\prime 2}-\kappa \rho \rho^{\prime \prime}\right) \boldsymbol{I}+\kappa \rho^{\prime 2} \boldsymbol{e}_{\zeta} \otimes \boldsymbol{e}_{\zeta}$ where $\boldsymbol{e}_{\zeta}$ denotes the unit vector normal to the interface, $\rho^{\prime}$ the partial derivative $\rho^{\prime}=\partial_{\zeta} \rho$ and similarly $\rho^{\prime \prime}=\partial_{\zeta}^{2} \rho=\Delta \rho$. By using the 
normal component of the zeroth order momentum equation (28), it is next obtained that $\bar{p}^{0}+\frac{1}{2} \kappa \rho^{\prime 2}-\kappa \rho \rho^{\prime \prime}$ is a constant denoted by $p^{\infty}=\bar{p}^{0}+\frac{1}{2} \kappa \rho^{\prime 2}-\kappa \rho \rho^{\prime \prime}$, which represents the constant pressure in the bulk phases far from the interface. After some algebra, the resulting zeroth order pressure tensor is of the form

$$
\overline{\mathcal{P}}=\left(p^{\infty}-\kappa \rho^{\prime 2}\right)\left(\boldsymbol{I}-\boldsymbol{e}_{\zeta} \otimes \boldsymbol{e}_{\zeta}\right)+p^{\infty} \boldsymbol{e}_{\zeta} \otimes \boldsymbol{e}_{\zeta}
$$

so that normal to the interface the effective pressure is constant equal to $p^{\infty}$ whereas there are attractive forces tangential to the interface and the corresponding energy $\int_{-\infty}^{\infty} \kappa \rho^{\prime 2} \mathrm{~d} \zeta$ is interpreted as surface tension [22,23].

For such interfaces, only the perturbed pressure $\widetilde{p}^{0}$ plays a role in the tangential momentum conservation equation and not the perturbed density $\widetilde{\rho}$. The zeroth order energy conservation equation in terms of the enthalpy $h=h^{0}$ also takes the simplified form

$$
\rho \partial_{t} h+\rho \boldsymbol{v} \nabla \cdot h+\nabla \cdot \mathcal{Q}=\partial_{t} \bar{p}^{0}+\boldsymbol{v} \cdot \nabla \bar{p}^{0}
$$

with a term $v \cdot \nabla \bar{p}^{0}$ that is not anymore a priori negligible as for standard fluids.

\subsection{Strained flows}

We investigate in this section self-similar solutions of the diffuse interface multicomponent equations in the small Mach number limit. These structures are typical of stagnation point flows or strained flows and will be used to investigate pseudo-vaporisation fronts as well as diffusion flames in the following sections. The spatial coordinates are denoted by $(\xi, \zeta)$, where $\xi$ is a transverse coordinate and $\zeta$ a normal coordinate, and the components of the velocity vector are denoted by $\boldsymbol{v}=(u, v)^{t}$. The typical geometry is either two-dimensional or axisymmetric. The solution is assumed to have the following self-similar structure:

$$
\begin{aligned}
& T=T(t, \zeta), \quad \rho=\rho(t, \zeta), \quad \bar{p}^{0}=\bar{p}^{0}(t, \zeta), \\
& u=\xi \tilde{u}(t, \zeta), \quad v=v(t, \zeta), \quad \mathrm{y}_{i}=\mathrm{y}_{i}(t, \zeta), \\
& \widetilde{p}^{0}=-\frac{1}{2} J \xi^{2}+\widehat{p}^{0}(t, \zeta)
\end{aligned}
$$

where $J$ is a reduced pressure gradient. We then obtain from (23) and (24) that the dissipative heat and mass fluxes are in the normal direction: $\mathcal{J}_{i}=\mathcal{J}_{i} \boldsymbol{e}_{\zeta}, i \in \mathfrak{S}$, and $\boldsymbol{q}=q \boldsymbol{e}_{\zeta}$, where $\boldsymbol{e}_{\zeta}$ denotes the base vector in the normal direction and $\mathcal{J}_{i}(t, \zeta), i \in \mathfrak{S}$, and $q(t, \zeta)$ are the normal flux components. Substituting these expressions in the governing equations in the small number limit, the normal momentum equation is found to uncouple from the others whereas the tangential momentum equation has all its terms proportional to $\xi$. The resulting system is a boundary value problem involving only the normal coordinate $\zeta$ in the form

$$
\begin{gathered}
\partial_{t} \rho+2^{\delta} \rho \tilde{u}+\partial_{\zeta}(\rho v)=0, \\
\rho \partial_{t} \mathbf{y}_{i}+\rho v \partial_{\zeta} y_{i}+\partial_{\zeta} \mathcal{J}_{i}=m_{i} \omega_{i}, \quad i \in \mathfrak{S}, \\
\rho \partial_{t} \tilde{u}+\rho \tilde{u}^{2}+\rho v \partial_{\zeta} \tilde{u}-J+\partial_{\zeta}\left(\eta \partial_{\zeta} \widetilde{u}\right)=0, \\
\rho \partial_{t} h+\rho v \partial_{\zeta} h-v \partial_{\zeta} \bar{p}^{0}+\partial_{\zeta}\left(\kappa \rho\left(2^{\delta} \tilde{u}+\partial_{\zeta} v\right) \partial_{\zeta} \rho\right)+\partial_{\zeta} q=0,
\end{gathered}
$$


where $\delta$ is a geometric factor with $\delta=0$ in a two-dimensional geometry and $\delta=1$ for axisymmetric flows. The dissipative normal mass and heat fluxes are given by (23) and (24) in their thermodynamic form:

$$
\begin{gathered}
\mathcal{J}_{i}=-\sum_{j \in \mathfrak{S}} L_{i j} \partial_{\zeta}\left(\frac{g_{j}^{0}}{T}\right)-L_{i e} \partial_{\zeta}\left(\frac{-1}{T}\right), \quad i \in \mathfrak{S}, \\
q=-\sum_{i \in \mathfrak{S}} L_{e i} \partial_{\zeta}\left(\frac{g_{i}^{0}}{T}\right)-L_{e e} \partial_{\zeta}\left(\frac{-1}{T}\right)
\end{gathered}
$$

and it has been established in Section 3.1 that the pressure $\bar{p}^{0}$ is such that

$$
\bar{p}^{0}=p^{\infty}-\frac{1}{2} \kappa\left(\partial_{\zeta} \rho\right)^{2}+\kappa \rho \partial_{\zeta}^{2} \rho
$$

where $p^{\infty}$ denotes the constant ambient pressure far from the interface. The boundary conditions are typically of the form

$$
\begin{gathered}
T(-\infty)=T^{-}, \quad T(+\infty)=T^{+}, \\
\mathrm{y}_{i}(-\infty)=\mathrm{y}_{i}^{-}, \quad \mathrm{y}_{i}(+\infty)=\mathrm{y}_{i}^{+}, \\
\tilde{u}(-\infty)=\alpha \sqrt{\rho^{+} / \rho^{-}}, \quad \tilde{u}(+\infty)=\alpha,
\end{gathered}
$$

where the superscript ${ }^{+}$is associated with the gas-like fluid coming from the positive side on the right, the superscript ${ }^{-}$with the condensed-like fluid coming from the negative side on the left. The imposed strain rate $\alpha$ is related to the pressure gradient by the relation

$$
\alpha=\left(J / \rho^{+}\right)^{1 / 2}
$$

and the stagnation plane is located for convenience at the origin by

$$
v(0)=0
$$

Thickening an interface may generally modify surface tension forces. Surface tension is typically of the form $\int_{-\infty}^{\infty} \kappa\left(\partial_{\zeta} \rho\right)^{2} \mathrm{~d} \zeta$ so that it scales as $\mathcal{O}(\sqrt{\kappa})$ in the subcritical domain with an interface thickness $\mathcal{O}(\sqrt{\kappa})$ and as $\mathcal{O}(\kappa)$ in the supercritical domain with an interface thickness $\mathcal{O}(1)$. There is thus a tradeoff between grid limitation and the resulting artificially increased surface tension. However, for such strained flames the surface tension forces do not significantly modify the bulk phase properties. Indeed, with planar interfaces, there are no curvature effects and the pressure $\bar{p}^{0}$ in both phases far from the vaporising front is identical and equal to the ambient pressure $p^{\infty}$. Essentially, the local pressure $\bar{p}^{0}$ is only modified near the pseudo-vaporising zone.

\subsection{Bulk thermodynamics}

The thermodynamics of the bulk phase fluid must be valid over a wide range of pressure and temperature and is built from the Soave-Redlich-Kwong cubic equation of state using 
compatibility with perfect gases at low densities [39-45]. Such thermodynamics are written in the small Mach number limit with the pressure given by its zeroth order value $\bar{p}^{0}$. The Soave-Redlich-Kwong equation of state $[39,40]$ is of the form

$$
\bar{p}^{0}=\sum_{i \in \mathfrak{S}} \frac{\mathrm{y}_{i}}{m_{i}} \frac{\rho R T}{1-\rho b}-\frac{\rho^{2} a}{1+\rho b},
$$

where $R$ denotes the perfect gas constant, and $a$ and $b$ the attractive and repulsive parameters, respectively. The parameters $a\left(\mathrm{y}_{1}, \ldots, \mathrm{y}_{n}, T\right)$ and $b\left(\mathrm{y}_{1}, \ldots, \mathrm{y}_{n}\right)$ are evaluated with the van der Waals mixing rules $a=\sum_{i, j \in \mathfrak{S}} \mathrm{y}_{i} \mathrm{y}_{j} \alpha_{i} \alpha_{j}$ and $b=\sum_{i \in \mathfrak{S}} \mathrm{y}_{i} b_{i}$. The purecomponent parameters $\alpha_{i}(T)$ and $b_{i}$ are evaluated using either critical data for stable species or interaction potential parameters for unstable species [9]. The validity of the equation of state (42) and of the mixing rules have been carefully studied by comparison with NIST data by Congiunti et al. [42] and with the results of Monte Carlo simulations by Colonna and Silva [43] and Cañas-Marín et al. [44,45]. Good agreement has also been achieved with experimental hydrogen/nitrogen stability diagrams at high pressure and low temperature [9].

Once a pressure law (42) is given there exists a unique corresponding Gibbsian thermodynamics compatible at low densities with that of perfect gases [41]. For the SoaveRedlich-Kwong equation of state it is possible to evaluate analytically the corresponding thermodynamic properties $[7,41]$, which are found in the form

$$
\begin{gathered}
e^{0}=\sum_{i \in \mathfrak{S}} \mathrm{y}_{i} e_{i}^{\mathrm{pg}}+\left(T \partial_{T} a-a\right) \frac{\ln (1+\rho b)}{b}, \\
s^{0}=\sum_{i \in \mathfrak{S}} \mathrm{y}_{i} s_{i}^{\mathrm{pg} I}-\sum_{i \in \mathfrak{S}} \frac{\mathrm{y}_{i} R}{m_{i}} \ln \left(\frac{\rho_{i} R T}{m_{i}(1-\rho b) p^{\mathrm{st}}}\right)+\partial_{T} a \frac{\ln (1+\rho b)}{b},
\end{gathered}
$$

where $e_{i}^{\mathrm{pg}}$ denotes the perfect gas specific energy of the $i$ th species and $s_{i}^{\mathrm{pg} I}$ the perfect gas specific entropy of the $i$ th species at the standard atmospheric pressure $p^{\text {st }}$.

A fundamental issue is then that of thermodynamic stability. The entropy of a stable isolated homogeneous system should indeed be a concave function of its thermodynamic variable $\gamma=\left(v, e^{0}, \mathrm{y}_{1}, \ldots, \mathrm{y}_{n}\right)^{t}$ where $v=1 / \rho$ denotes the volume per unit mass. Whenever this is not the case, the system loses its homogeneity and splits between two or more phases in order to reach equilibrium. The Hessian matrix of entropy $s^{0}$ with respect to the thermodynamic variable $\gamma$ must therefore be negative semi-definite with the nullspace spanned $N\left(\partial_{\gamma \gamma}^{2} s^{0}\right)=\mathbb{R} \gamma$ associated with homogeneity and this can be shown to be equivalent to the conditions [41]

$$
\partial_{T} e^{0}>0, \quad \partial_{\rho} \bar{p}^{0}>0, \quad\left(\partial_{\mathrm{yy}}^{2} g^{0}\right)_{T, \bar{p}^{0}} \geq 0, \quad N\left(\left(\partial_{\mathrm{yy}}^{2} g^{0}\right)_{T, \bar{p}^{0}}\right)=\mathbb{R} \mathbf{y}
$$

The first condition $\partial_{T} e^{0}>0$ corresponds to thermal stability, the second condition $\partial_{\rho} \bar{p}^{0}>0$ to mechanical stability and the third condition $\left(\partial_{\mathrm{yy}}^{2} g^{0}\right)_{T, \bar{p}^{0}} \geq 0$ with $N\left(\left(\partial_{\mathrm{yy}}^{2} g^{0}\right)_{T, \bar{p}^{0}}\right)=\mathbb{R y}$ to chemical stability. The mechanical and chemical stability criteria can also be combined into the property that the matrix $\Lambda$, defined by

$$
\Lambda=\left(\partial_{\mathrm{yy}}^{2} e^{0}\right)_{T, \rho} / T-\left(\partial_{\mathrm{yy}}^{2} s^{0}\right)_{T, \rho}
$$


is positive definite [41]. Since the thermal stability condition is generally satisfied with the SRK equation of state $[9,41,46]$, thermodynamic stability may therefore be investigated by a spectral analysis of the matrix $\Lambda$. Note that when a multicomponent mixture reaches a pure species state the thermodynamic instability limit is then given by the mechanical stability of that species [41].

Finally note that bulk fluid thermodynamics plays a fundamental role in the state law, the equilibrium diagrams, the energy equation, the chemical reaction rates as well as the nonideal diffusion fluxes; the bulk fluid thermodynamics has not, therefore, been artificially modified in order to decrease the surface tension resulting from second gradient theory [23].

\subsection{Dissipative transport fluxes}

The evaluation of multicomponent transport fluxes requires the mass and heat transport coefficient matrix $L$ of size $n+1$ to be evaluated. This matrix can be written in the form $[9,46]$

$$
L=\left(\begin{array}{cc}
\mathcal{D} & \mathcal{D} h \\
(\mathcal{D} h)^{t} & \lambda T^{2}+\langle\mathcal{D} \mathfrak{h}, \hat{h}\rangle
\end{array}\right)
$$

where $\mathcal{D}$ denotes a square matrix of size $n, f$ a vector of size $n, \lambda$ the thermal conductivity, and $\langle$,$\rangle the Euclidean scalar product. The matrix coefficients \mathcal{D}_{i j}, i, j \in \mathfrak{S}$, and the vector $f_{i}, i \in \mathfrak{S}$, are given by

$$
\mathcal{D}_{i j}=\rho \mathrm{y}_{i} \mathrm{y}_{j} \frac{m}{R} D_{i j}, \quad \digamma_{i}=h_{i}+R T \frac{\tilde{\chi}_{i}}{m_{i}}
$$

where $D_{i j}, i, j \in \mathfrak{S}$, denote the species multicomponent diffusion coefficients, $h_{i}, i \in \mathfrak{S}$, the species specific enthalpies, and $\tilde{\chi}_{i}, i \in \mathfrak{S}$, the species reduced thermal diffusion ratios $[9,46]$. The multicomponent diffusion coefficients $D_{i j}$ are obtained with an approximate inversion [38,47-51] using

$$
D=(\Delta+\mathrm{y} \otimes \mathrm{y})^{-1}-\mathrm{u} \otimes \mathrm{u},
$$

where $y$ denotes the mass fraction vector, $\mathrm{u}=(1, \ldots, 1)^{t}$ the vector with unit components, $\Delta$ the Stefan-Maxwell matrix

$$
\Delta_{k k}=\sum_{l \neq k} \frac{\mathbf{x}_{k} \mathbf{x}_{l}}{\mathcal{D}_{k l}^{\text {bin }}}, \quad \Delta_{k l}=-\frac{\mathbf{x}_{k} \mathbf{x}_{l}}{\mathcal{D}_{k l}^{\text {bin }}}, \quad k \neq l,
$$

$\mathrm{x}_{k}$ the mole fraction of the $k$ th species, and $\mathcal{D}_{k l}^{\text {bin }}$ the binary coefficient for the species pair $(k, l), k \neq l$. The binary diffusion coefficients $\mathcal{D}_{k l}^{\text {bin }}, k, l \in \mathfrak{S}, k \neq l$, are evaluated from the kinetic theory of a mixture of hard spheres $[9,52,53]$ in the form $\mathcal{D}_{k l}^{\text {bin }}=\mathcal{D}_{k l}^{\text {bin pg }} / \Upsilon_{k l}$, $k, l \in \mathfrak{S}, k \neq l$, where $\mathcal{D}_{k l}^{\text {bin pg }}$ denotes the perfect gas binary diffusion coefficient and $\Upsilon_{i j}$ the steric factor

$$
\Upsilon_{i j}=1+\sum_{k \in \mathfrak{S}} \frac{\pi \mathfrak{n}_{k}}{12}\left(8\left(\sigma_{i k}^{3}+\sigma_{j k}^{3}\right)-6\left(\sigma_{i k}^{2}+\sigma_{j k}^{2}\right) \sigma_{i j}-3\left(\sigma_{i k}^{2}-\sigma_{j k}^{2}\right)^{2} \sigma_{i j}^{-1}+\sigma_{i j}^{3}\right) .
$$




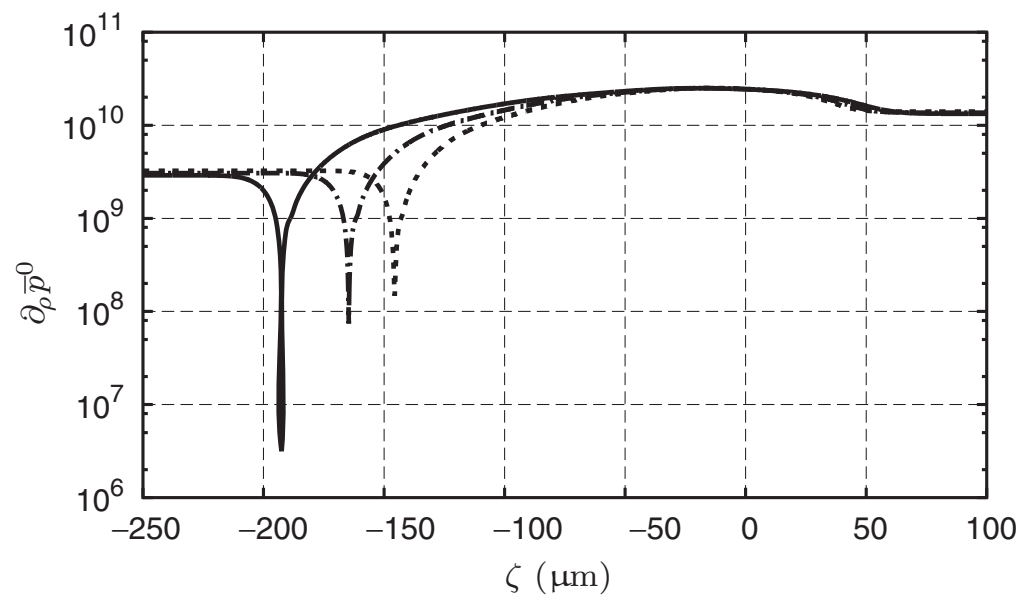

Figure 1. The derivative $\partial_{\rho} \bar{p}^{0}$ in a diffusion flame: $\_p^{\infty}=51$ bar; $\ldots-p^{\infty}=70$ bar; $\ldots$. $p^{\infty}=$ 90 bar.

In this expression, $\sigma_{i j}$ denotes the collision diameter for the species pair $(i, j), \mathfrak{n}_{k}=\rho_{k} \mathcal{N} / m_{k}$ the particle number of the $k$ th species per unit volume and $\mathcal{N}$ Avogadro's number. The viscosity and thermal conductivity are evaluated from the correlations of Ely and Hanley [54] or Chung et al. [9,55] and the thermal diffusion ratios are evaluated as for ideal gases [9]. The properties of the matrix $L$ are derived from the properties of the matrix $D=\left(D_{i j}\right)_{i, j \in \mathfrak{S}}$, which is symmetric positive semi-definite having nullspace $N(D)=\mathbb{R y}[38,47-51]$.

Some care must be taken, however, in order to evaluate the species specific enthalpies $h_{i}=\left(\partial_{y_{i}} h\right)_{T, \bar{p}^{0}, \mathrm{y}_{l}}, i \in \mathfrak{S}$, in the presence of mechanical critical points where $\partial_{\rho} \bar{p}^{0}=0$. We indeed have the identity

$$
h_{k}=\left(\partial_{\mathrm{y}_{k}} h\right)_{T, \bar{p}^{0}, \mathrm{y}_{l}}=\left(\partial_{\mathrm{y}_{k}} h\right)_{T, \rho, \mathrm{y}_{l}}-\left(\partial_{\rho} h\right)_{T, \mathrm{y}_{l}} \frac{\left(\partial_{\mathrm{y}_{k}} \bar{p}^{0}\right)_{T, \rho, \mathrm{y}_{l}}}{\left(\partial_{\rho} \bar{p}^{0}\right)_{T, \mathrm{y}_{l}}}
$$

so that, at a mechanical thermodynamically unstable point where $\left(\partial_{\rho} \bar{p}^{0}\right)_{T, \mathrm{y}_{l}}=0$, the species specific enthalpies $h_{k}, k \in \mathfrak{S}$, are exploding and thus some components of the matrix $L$ are also exploding. This is expected behaviour, since it is known that singularities in the transport coefficients may arise at critical points [56,57].

Anticipating the numerical simulations of flame structures, Figure 1 presents the partial derivative $\left(\partial_{\rho} \bar{p}^{0}\right)_{T, y_{l}}$ as a function of the normal coordinate $\zeta$ in an oxygen/hydrogen strained diffusion flame with $\kappa=10^{-2} \mathrm{~g}^{-1} \mathrm{~cm}^{7} \mathrm{~s}^{-2}$ for the three values $p^{\infty}=51 \mathrm{bar}, p^{\infty}=70 \mathrm{bar}$ and $p^{\infty}=90$ bar of the ambient pressure. We note that the minimum value of the derivative $\left(\partial_{\rho} \bar{p}^{0}\right)_{T, \mathrm{y}_{l}}$ is gradually decreasing towards zero when the ambient pressure is decreased towards the oxygen critical pressure $p_{c}=50.43$ bar with a local mixture state approaching the mechanical stability limit near the pseudo-vaporising zone. The corresponding specific enthalpy of water $h_{\mathrm{H}_{2} \mathrm{O}}(\zeta)$ presented in Figure 2 then gradually explodes as the local state of the mixture reaches the mechanical stability boundary in agreement with (45).

It is therefore necessary to control the size of the vector $f$ making use of the fact that in the cold part of an oxygen/hydrogen diffusion flame there is mainly oxygen. To this aim, 


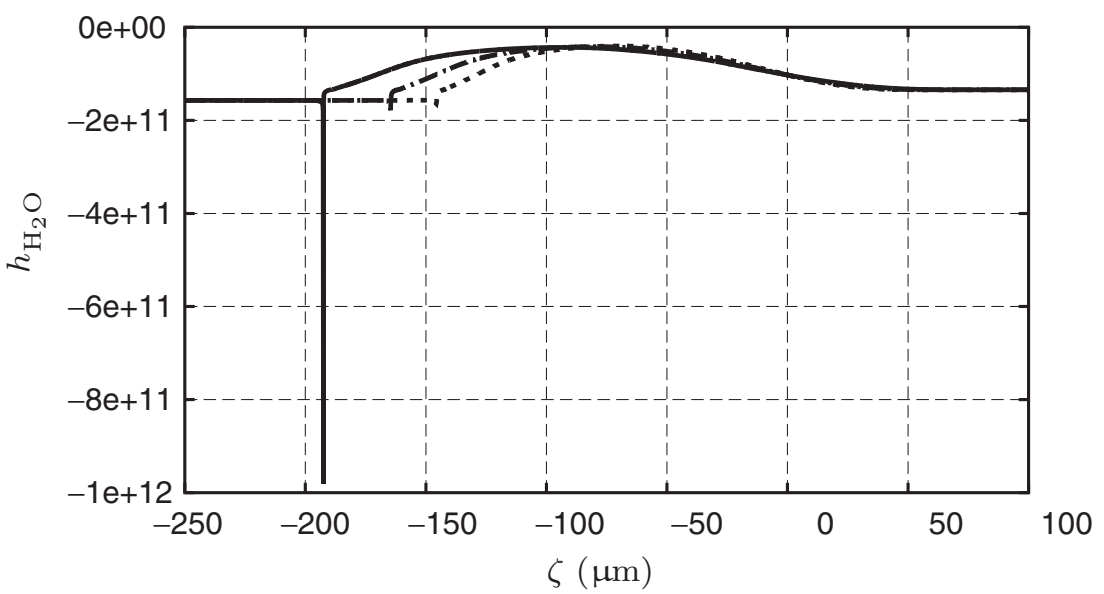

Figure 2. Water specific enthalpy $h_{\mathrm{H}_{2} \mathrm{O}}$ in a diffusion flame: $-p^{\infty}=51$ bar; $-.-p^{\infty}=70$ bar; $\ldots p^{\infty}=90$ bar.

the derivative $\left(\partial_{\mathbf{y}_{k}} \bar{p}^{0}\right)_{T, \rho, \mathbf{y}_{l}}$ is rewritten in the form

$$
\left(\partial_{\mathrm{y}_{k}} \bar{p}^{0}\right)_{T, \rho, \mathrm{y}_{l}}=\frac{\rho m}{m_{k}}\left(\partial_{\rho} \bar{p}^{0}\right)_{T, \mathrm{y}_{l}}+\mathcal{R}_{k}
$$

where the residual $\mathcal{R}_{k}$ is given by

$\mathcal{R}_{k}=\left\{\frac{R T}{(v-b)^{2}}+\frac{m a}{v(v+b)^{2}}\right\} \sum_{j \in \mathfrak{S}} \mathrm{y}_{j}\left(\frac{b_{k}}{m_{j}}-\frac{b_{j}}{m_{k}}\right)+\frac{2 m}{v(v+b)} \sum_{j, l \in \mathfrak{S}} \mathrm{y}_{j} \mathrm{y}_{l} \alpha_{l}\left(\frac{\alpha_{j}}{m_{k}}-\frac{\alpha_{k}}{m_{j}}\right)$.

The interest of this formulation (46) is that $\mathcal{R}_{k}$ vanishes for the pure species state $\mathrm{y}_{k}=1$ and $\mathrm{y}_{l}=0, l \in \mathfrak{S}, l \neq k$. The species enthalpies are then written in the modified form $\widetilde{h}_{k}$, $k \in \mathfrak{S}$, with

$$
\widetilde{h}_{k}=\left(\partial_{\mathrm{y}_{k}} h\right)_{T, \rho, \mathrm{y}_{l}}-\frac{\rho m}{m_{k}}\left(\partial_{\rho} h\right)_{T, \mathrm{y}_{l}}-\frac{\mathcal{R}_{k}}{\mathfrak{f}\left(\left(\partial_{\rho} \bar{p}^{0}\right)_{T, \mathrm{y}_{l}}\right)}\left(\partial_{\rho} h\right)_{T, \mathrm{y}_{l}},
$$

where $\mathfrak{f}$ is a smooth approximation of the function $\mathfrak{f}(x)=\max (\mathrm{m}, x)$ for a positive constant $\mathrm{m}>0$ which depends on the mixture under consideration. These modified enthalpies are such that $\widetilde{h_{k}}=h_{k}$ away from the vaporising zone, as well as when the $k$ th species is the only one present, and remains always bounded. The points where the matrix $L$ is effectively modified thus only concern zones of the flames where there is essentially only oxygen and where temperature is also nearly constant so that the stabilisation of the matrix $L$ does not significantly modify the physics involved. For similar reasons we did not include in the model the complex behaviour of transport coefficients near critical points.

Remark 2: The traditional form of dissipative fluxes may be written $[9,46]$

$$
\mathcal{J}_{i}=-\sum_{j \in \mathfrak{S}} \rho \mathbf{y}_{i} D_{i j}\left(d_{j}+\mathbf{x}_{j} \tilde{\chi}_{j} \partial_{\zeta} \ln T\right) \quad i \in \mathfrak{S},
$$




$$
q=-\lambda \partial_{\zeta} T+\sum_{i \in \mathfrak{S}}\left(h_{i}+R T \frac{\tilde{\chi}_{i}}{m_{i}}\right) \mathcal{J}_{i},
$$

where $d_{j}, j \in \mathfrak{S}$, are the species diffusion driving force in the normal direction. These diffusion driving forces are defined by $d_{j}=\mathbf{x}_{j}\left(\partial_{\zeta} \mu_{j}\right)_{T}, j \in \mathfrak{S}$, where the derivative of the reduced chemical potential $\left(\partial_{\zeta} \mu_{j}\right)_{T}$ is taken at constant temperature and with $\mu_{j}$ written as a function of temperature, pressure and mole fractions $\mu_{j}\left(T, \bar{p}^{0}, \mathbf{x}_{1}, \ldots, \mathbf{x}_{n}\right)$. From the Gibbs relation we further obtain that $\mathrm{x}_{j} \partial_{\zeta} \mu_{j}=d_{j}-\left(\mathrm{x}_{j} m_{j} h_{j} / R T^{2}\right) \partial_{\zeta} T$ so that $d_{j}$ may be written $[9,46]$

$$
d_{j}=\frac{\mathbf{x}_{j} m_{j} v_{j}}{R T} \partial_{\zeta} \bar{p}^{0}+\sum_{l \in \mathfrak{S}} \Gamma_{j l} \partial_{\zeta} \mathbf{x}_{l}, \quad j \in \mathfrak{S},
$$

where $v_{j}, j \in \mathfrak{S}$, denote the species partial volume per unit mass of the $j$ th species, $\Gamma_{j l}$ the thermodynamic coefficients $\Gamma_{j l}=\mathrm{x}_{j}\left(\partial_{\mathbf{x}_{l}} \mu_{j}\right)_{T, \bar{p}^{0}, \mathbf{x}_{k}}, j, l \in \mathfrak{S}$, and the matrix $\Gamma=$ $\left(\Gamma_{i j}\right)_{i, j \in \mathfrak{S}}$ reduces to the identity matrix for ideal gases. Since pressure is held constant in the partial derivations, we deduce that the species volume per unit mass $v_{j}, j \in \mathfrak{S}$, and the thermodynamic coefficients $\Gamma_{i j}, i, j \in \mathfrak{S}$, are exploding when $\left(\partial_{\rho} \bar{p}^{0}\right)_{T, \mathrm{y}_{l}}=0$ like the species enthalpies per unit mass $h_{j}, j \in \mathfrak{S}$. Therefore, the traditional formulation of transport fluxes (47) and (48) is not recommended and the thermodynamical formulation (34) and (35) is to be preferred. Such thermodynamic form indeed eliminates the exploding quantities $d_{i}, v_{i}, i \in \mathfrak{S}, \Gamma_{i j}, i, j \in \mathfrak{S}$, from the species mass fluxes, as well as the specific enthalpies $h_{i}, i \in \mathfrak{S}$, from the expression for the heat flux.

\subsection{Numerical considerations}

The two-point boundary value problems (30)-(41) associated with strained diffuse interfaces are discretised by using finite differences on staggered grids. The normal velocity is discretised at midpoints whereas the other quantities are discretised at node points.

The numerical grids are adaptively refined and the resulting discrete nonlinear problems are solved using the damped Newton method and pseudo-unsteady iterations [58,59]. Continuation techniques are used to generate families of critical points or equilibrium states as well as vaporising fronts or flame structures [60].

The resulting flame model in one-dimensional and all calculations have been performed on single-processor computers with typically 2000 grid points distributed adaptively.

\section{Oxygen/oxygen interfaces}

We consider in this section an interface between cold liquid-like oxygen and hotter gaseouslike oxygen. In the subcritical domain, both liquid and gaseous phases are thermodynamically stable and we compute a liquid/gas diffuse interface, whereas in the supercritical domain there is only one thermodynamically stable phase at stiff density gradients in the pseudo-vaporisation zone. Only steady-state interfaces are considered and the corresponding equations are obtained by letting $\partial_{t}=0$ in (30)-(33).

The numerical simulations first concern interfaces between cold dense oxygen and gaseous oxygen. We present the structure of typical interfaces at subcritical as well as supercritical conditions. Subcritical interfaces are found to be diffuse due to capillarity 


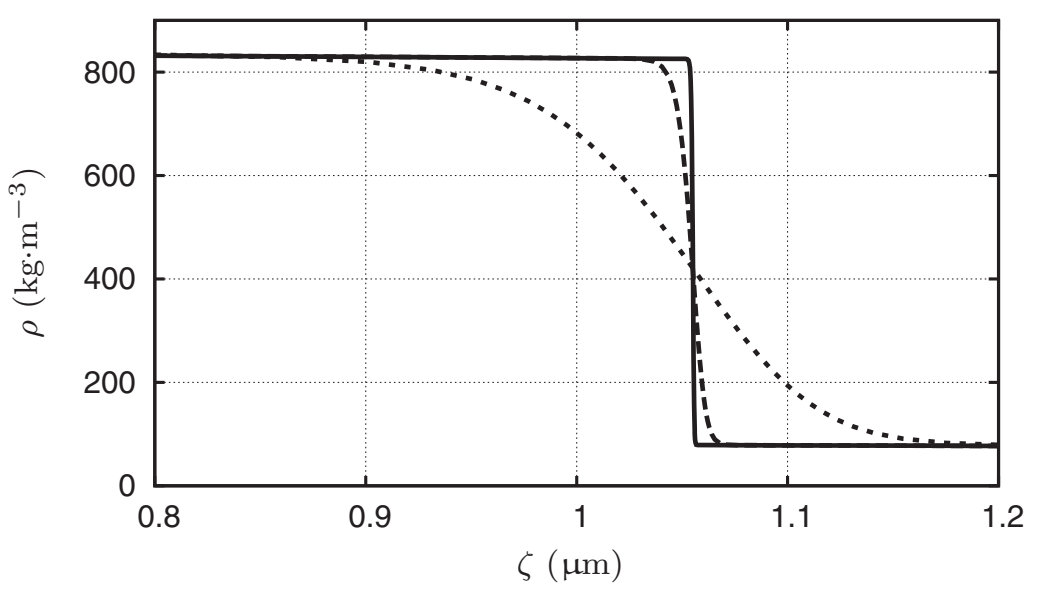

Figure 3. Density $\rho$ in a diffuse oxygen interface at $p^{\infty}=20$ bar: $\_\kappa=10^{-6} ;--\kappa=10^{-4} ; \ldots$ $\kappa=10^{-2}$.

whereas supercritical interfaces are only weakly perturbed at large pressure. Phase diagrams also reveal how the unstable thermodynamic zones are crossed in the subcritical regime with the help of second gradient theory. We also investigate the interface thickness in terms of the pressure and the capillary coefficient $\kappa$ as well as the influence of pressure on the interface structure. In the subcritical regime, the interface thickness decreases towards zero with $\kappa$ whereas in the supercritical regime the diffuse interface thickness is close to that of the real interface obtained with $\kappa=0$. In the subcritical regime, there is also a natural scaling $\delta \sim \sqrt{\kappa}$ of the interface thickness arising from the definition of the van der Waals free energy.

\subsection{Structure of interfaces}

We consider a subcritical pressure of $p^{\infty}=20$ bar and a supercritical pressure of $p^{\infty}=$ 52.5 bar, the critical pressure of oxygen being $p_{c}=50.43$ bar. The supercritical pressure $p^{\infty}=52.5$ bar is chosen close to the critical point in order to illustrate the diffuse interface better. The strain rate $\alpha=1000 \mathrm{~s}^{-1}$ corresponds to a velocity decreasing from $100 \mathrm{~cm} \mathrm{~s}^{-1}$ to zero within $1 \mathrm{~mm}$ and is representative of strain rates in flames. The temperature in the cold stream on the negative side is taken to be $T^{-}=100 \mathrm{~K}$ and in the hot stream on the positive side $T^{+}=300 \mathrm{~K}$. For these vaporising or pseudo-vaporising interfaces, $\mathrm{O}_{2}$ is the only chemical species and no species equations are solved.

In Figure 3 is presented the mass density $\rho(\zeta)$ as a function of the front normal coordinate $\zeta$ near the $\mathrm{O}_{2}$ vaporising zone at the subcritical pressure $p^{\infty}=20$ bar for the three values of the capillary parameter $\kappa=10^{-6}, \kappa=10^{-4}$ and $\kappa=10^{-2} \mathrm{~g}^{-1} \mathrm{~cm}^{7} \mathrm{~s}^{-2}$. We observe a major impact of the capillary parameter $\kappa$ on the structure of the interface. The lower the value of $\kappa$, the sharper the interface and in the limit $\kappa \rightarrow 0$ the discontinuous solution with an infinitely thin interface is recovered. This is natural in the sense that such diffuse behaviour is the direct result of the van der Waals theory and of the presence of extra gradient terms in the free energy. In Figure 4 is presented the mass density $\rho(\zeta)$ as function of the front normal coordinate $\zeta$ near the $\mathrm{O}_{2}$ pseudo-vaporising zone at the supercritical pressure of $p^{\infty}=52.25$ bar for the three values of the capillary parameter $\kappa=0 \mathrm{~g}^{-1} \mathrm{~cm}^{7} \mathrm{~s}^{-2}, \kappa=10^{-4} \mathrm{~g}^{-1} \mathrm{~cm}^{7} \mathrm{~s}^{-2}$, and $\kappa=10^{-2} \mathrm{~g}^{-1} \mathrm{~cm}^{7} \mathrm{~s}^{-2}$. Of course the zero 


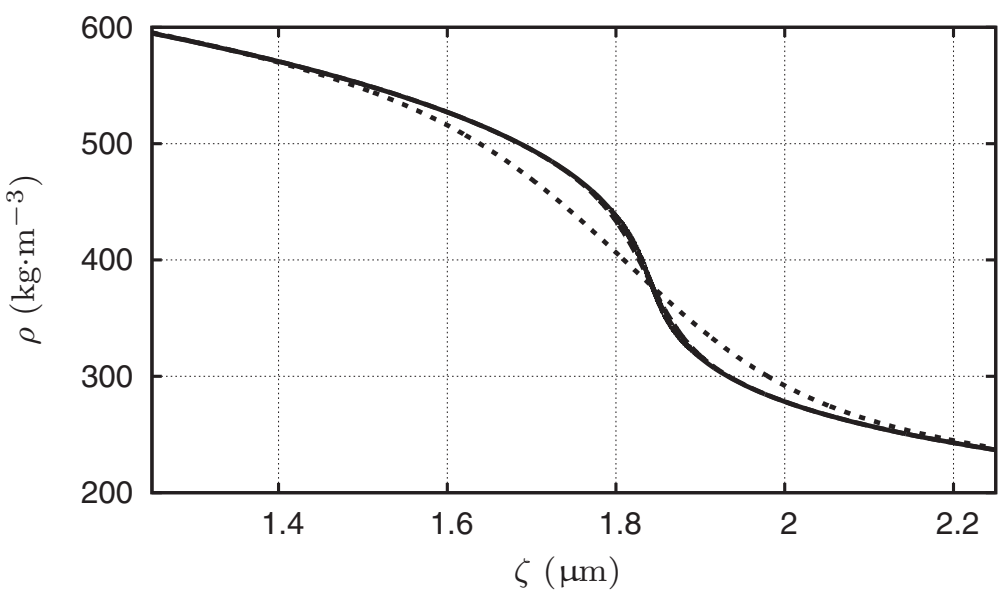

Figure 4. Density $\rho$ in a diffuse oxygen interface at $p^{\infty}=52.5$ bar: $\_\kappa=0 ; \ldots \kappa=10^{-4}$; _ . $\kappa=10^{-2}$.

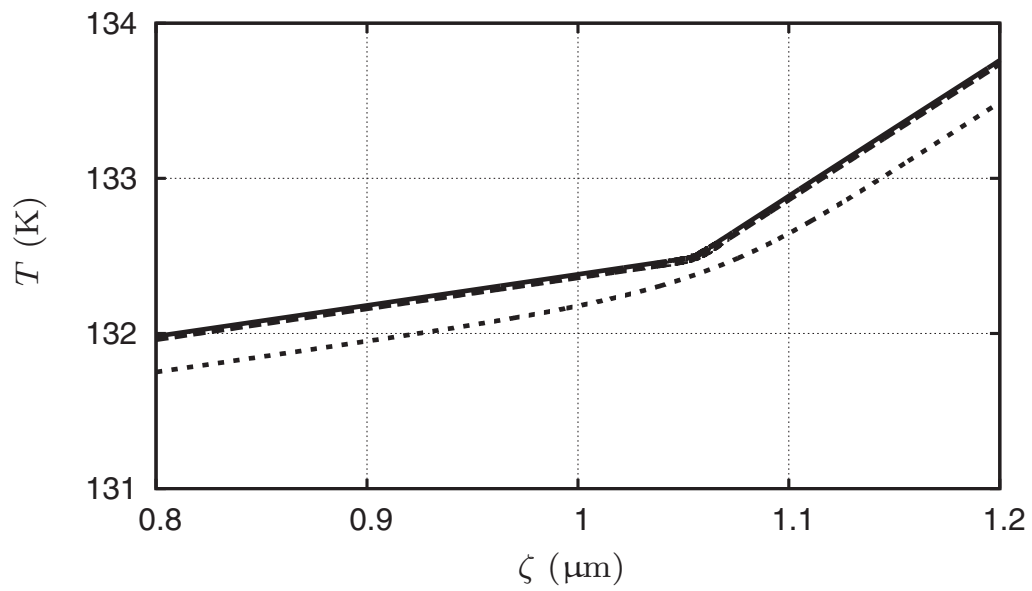

Figure 5. Temperature $T$ in a diffuse oxygen interface at $p^{\infty}=20$ bar: $\_\kappa=10^{-6} ;--\kappa=10^{-4}$; $\ldots \kappa=10^{-2}$.

value of the capillary parameter is allowed here since the pressure $p^{\infty}$ is supercritical. In this supercritical condition, we observe that the interface is naturally thick and only weakly influenced by the capillary terms.

The corresponding temperature profiles $T(\zeta)$ are presented in Figures 5 and 6 for ambient pressures of both $p^{\infty}=20$ and 52.5 bar, respectively. We observe that the temperature profiles are only weakly modified by the capillary coefficient $\kappa$. The interfaces or pseudointerfaces where the density gradient plays an important role are indeed thin and as a result the temperature is nearly constant in these zones, whereas in the remaining part of the domain the temperature is governed by convection and diffusion.

The corresponding local pressure profiles $\bar{p}^{0}(\zeta)$ near the $\mathrm{O}_{2}$ vaporising or pseudovaporising zones are presented in Figures 7 and 8 for ambient pressures of both $p^{\infty}=20$ and 52.5 bar, respectively. We observe an important difference between the subcritical and the supercritical situations. In the subcritical situation, in Figure 7, the pressure curve is 


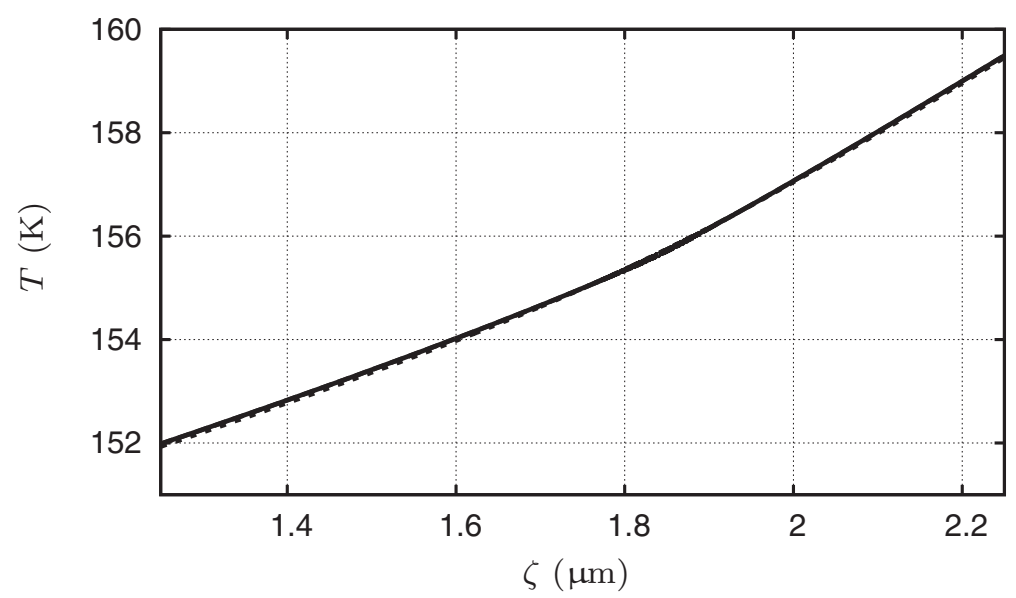

Figure 6. Temperature $T$ in a diffuse oxygen interface at $p^{\infty}=52.5$ bar: $\_\kappa=0 ;--\kappa=10^{-4}$; $\ldots \kappa=10^{-2}$.

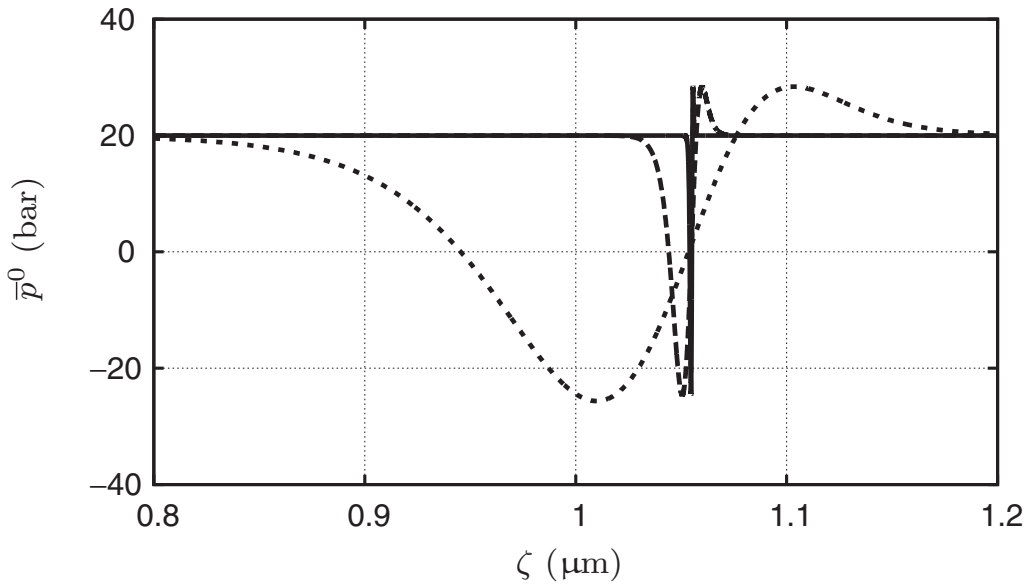

Figure 7. Pressure $\bar{p}^{0}$ in a diffuse oxygen interface at $p^{\infty}=20$ bar: $\_\kappa=10^{-6}$; $--\kappa=10^{-4}$; $\ldots \kappa=10^{-2}$.

strained differently depending of the capillary parameter $\kappa$ but is otherwise almost selfsimilar. This is not the case at supercritical pressure in Figure 8 where the local pressure variation in the interfacial region goes to zero with the capillary parameter $\kappa$.

The behaviour of the local pressure $\bar{p}^{0}$ at subcritical ambient pressure $p^{\infty}$ is due to the fact that the diffuse interface trajectory in the phase space $\left(\rho, \bar{p}^{0}\right)$ essentially follows an isothermal curve of the SRK equation of state at the interface. This is illustrated in Figure 9 where are presented the lines of equilibrium, the line of mechanical instability where an eigenvalue of the entropy Hessian is changing sign, the SRK isotherm at the interface temperature and the interface trajectory for the value of the capillary parameter $\kappa=10^{-6} \mathrm{~g}^{-1} \mathrm{~cm}^{7} \mathrm{~s}^{-2}$. The zone between the equilibrium line and the line of mechanical instability corresponds to metastable states and is grey in colour. The interface trajectory closely follows the SRK isotherm in the unstable zone in such a way that at subcritical pressure $p^{\infty}$ the local pressure profiles $\bar{p}^{0}(\zeta)$ are almost self-similar for various values of 


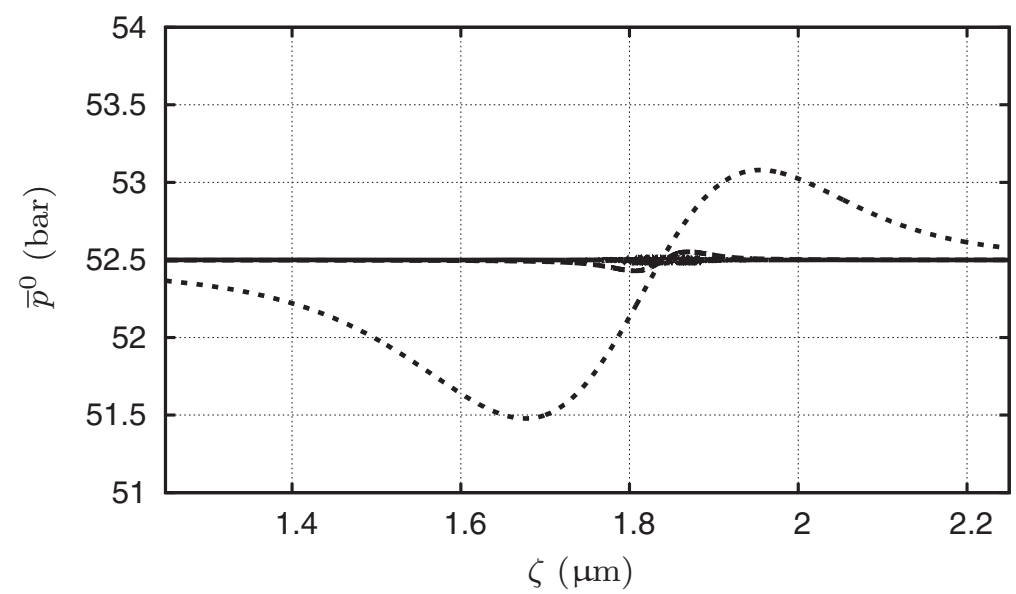

Figure 8. Pressure $\bar{p}^{0}$ in a diffuse oxygen interface at $p^{\infty}=52.5$ bar: $\_\kappa=0 ; \ldots \kappa=10^{-4}$; _ . $\kappa=10^{-2}$.

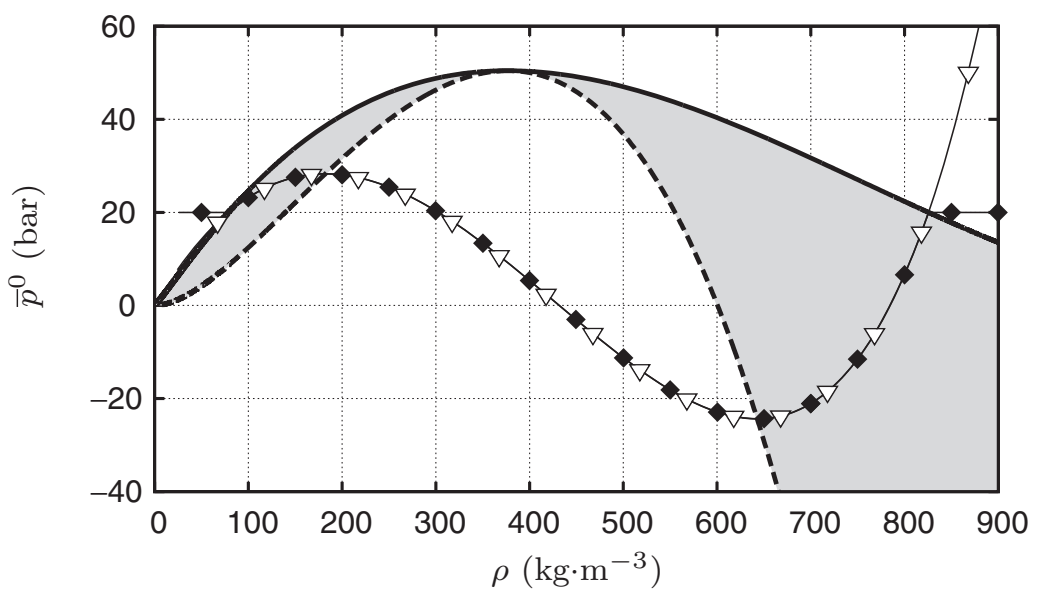

Figure 9. Phase space $\left(\rho, \bar{p}^{0}\right)$ for a diffuse oxygen interface at $p^{\infty}=20$ bar: __ equilibrium; - mechanical stability limit; $\nabla$ SRK isotherm; $\downarrow$ diffuse interface trajectory. The grey zone corresponds to metastable states.

the capillary coefficients $\kappa$. This also explains the negative values of $\bar{p}^{0}$ in the interface which are a direct consequence of the negative values of the pressure given by the SRK equation of state.

\subsection{Dependence on pressure}

A fundamental issue with diffuse interface models is the interface thickness $\delta$ as a function of pressure $p^{\infty}$ and of the capillary parameter $\kappa$. The thickness of the interface $\delta$ is defined 


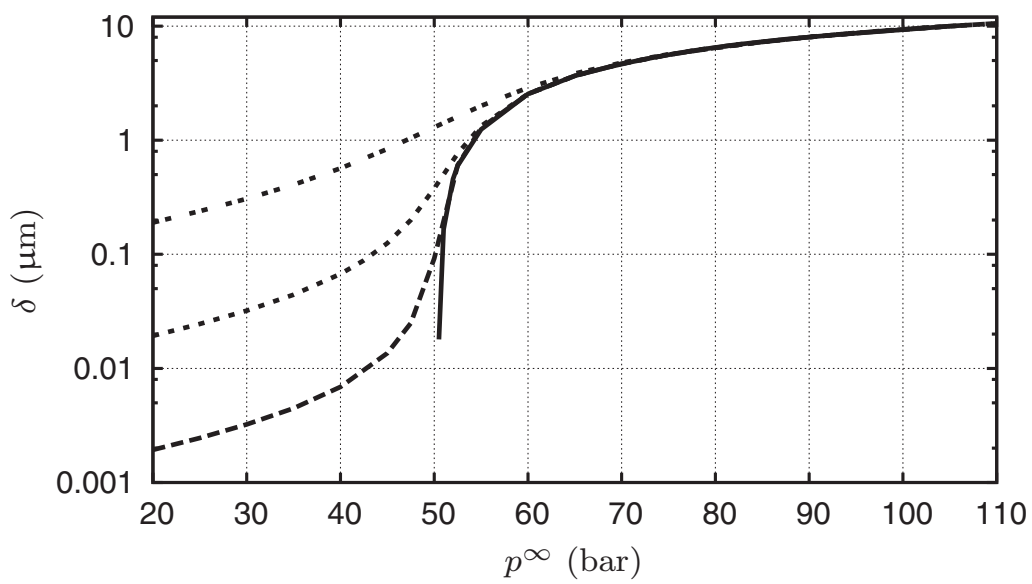

Figure 10. Thickness of oxygen diffuse interfaces: $\_\kappa=0 ; \ldots \kappa=10^{-6} ; \ldots \kappa=10^{-4} ; \ldots \ldots$ $\kappa=10^{-2}$.

here by

$$
\delta=\frac{\rho^{-}-\rho^{+}}{\max _{-\infty<\zeta<\infty}\left|\partial_{\zeta} \rho\right|},
$$

where $\rho^{-}$and $\rho^{+}$denote respectively the two extreme values of density at the left/dense and right/light boundaries, and $\max \left|\partial_{\zeta} \rho\right|$ is the maximum value of the gradient $\left|\partial_{\zeta} \rho(\zeta)\right|$ for $-\infty<\zeta<+\infty$.

The diffuse interface thickness $\delta$ is presented in Figure 10 in terms of the pressure $p^{\infty}$ and of the capillary parameter $\kappa$. The critical pressure of $\mathrm{O}_{2}$ is $p_{c}=50.43$ bar and Figure 10 illustrates the clear separation between the subcritical and the supercritical situations. In the subcritical regime, the interface thickness decreases with $\kappa$, whereas in the supercritical regime the diffuse interface thickness is close to that of the real interface with $\kappa=0$. Figure 10 also illustrates the natural scaling $\delta-\delta_{0} \sim \sqrt{\kappa}$ of the interface problem arising from the definition of the van der Waals free energy, where $\delta_{0}$ is the interface thickness when $\kappa=0$, so that $\delta \sim \sqrt{\kappa}$ in the subcritical region. In particular, the choice of a capillary parameter $\kappa$ in a numerical model may be guided by the size of the discretisation grid in order to represent density gradients in subcritical conditions properly.

In the next figures, we further investigate the dependence of oxygen vaporising fronts on the pressure $p^{\infty}$ for the value $\kappa=10^{-2} \mathrm{~g}^{-1} \mathrm{~cm}^{7} \mathrm{~s}^{-2}$ of the capillary parameter. This large value of the capillary parameter is made to illustrate how sharp the vaporisation fronts remain in subcritical conditions. The interface structure, however, is not fundamentally dependent on the precise value of the capillary parameter since in the subcritical domain the vaporisation front is essentially self-similar whereas in the supercritical domain the pseudo-vaporisation zone is only weakly perturbed as shown in Figure 10. In Figure 11 are presented the density profiles $\rho(\zeta)$, in Figure 12 the temperature profiles $T(\zeta)$ and in Figure 13 the local pressure profiles $\bar{p}^{0}(\zeta)$ for the ambient pressures $p^{\infty}=20,40$ and 60 bar. The very sharp structure of the vaporising fronts for subcritical interfaces is illustrated in Figures 11 and 13. The corresponding temperature profiles in Figure 12 also illustrate the importance of the latent heat in the subcritical regime associated with the fast variation of 


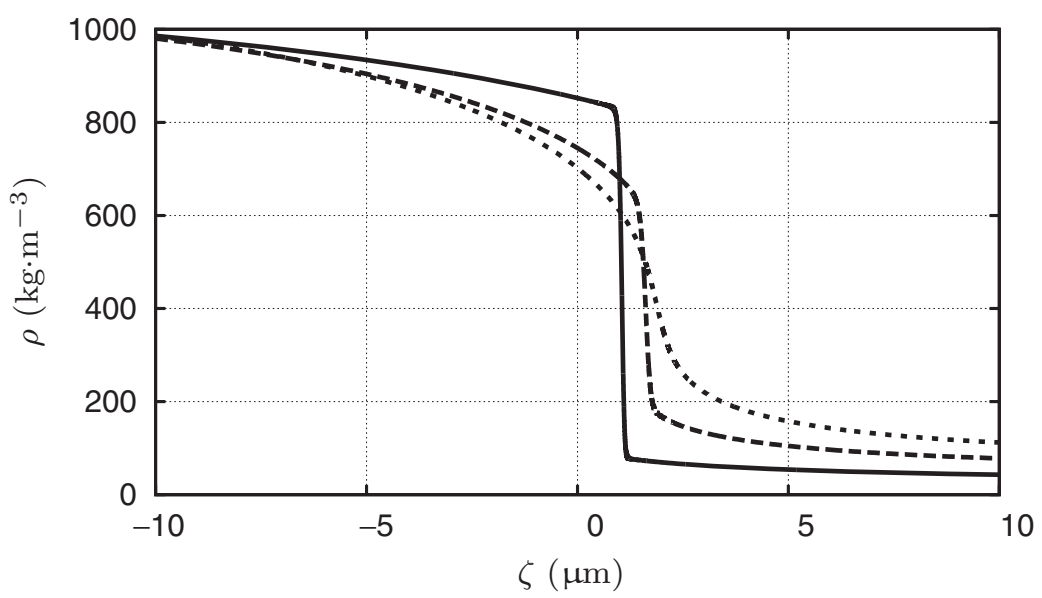

Figure 11. Density $\rho$ in a diffuse oxygen interface: __ 20 bar; __ 40 bar; _ _ _ 60 bar.

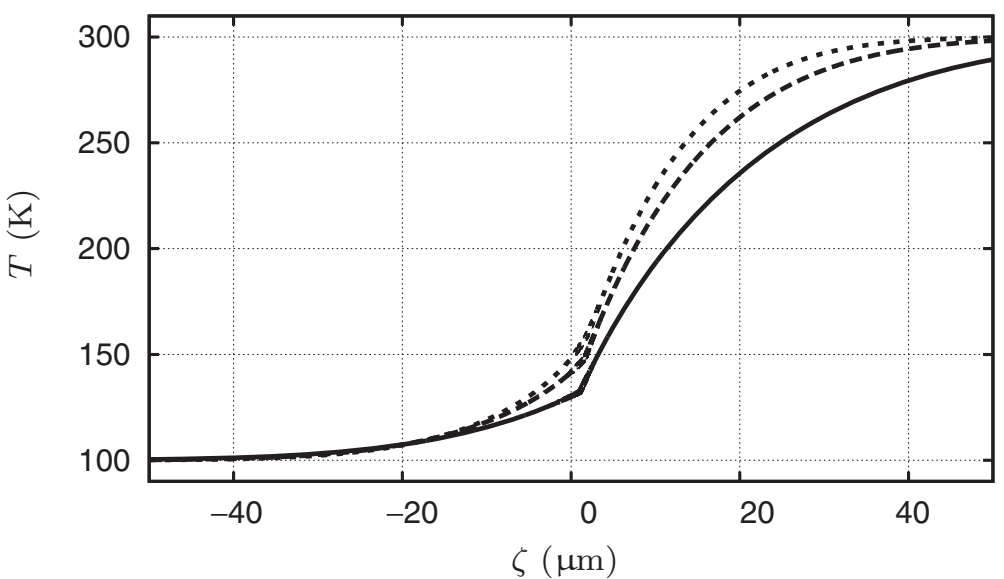

Figure 12. Local pressure $\bar{p}^{0}$ in a diffuse oxygen interface: __ 20 bar; _ _ 40 bar; . . 60 bar.

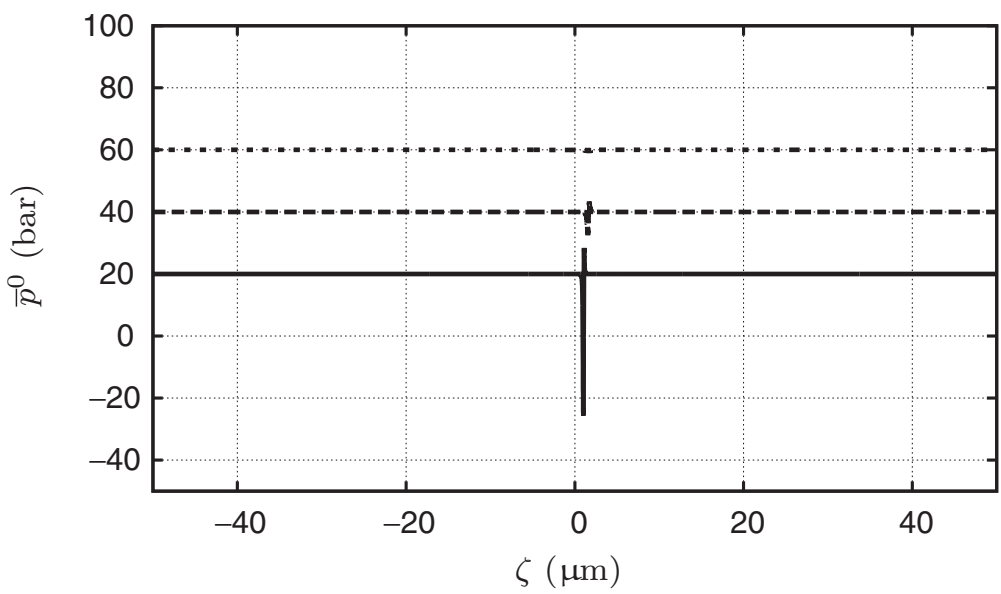

Figure 13. Pressure $\bar{p}^{0}$ in a diffuse oxygen interface: __ 20 bar; _ _ 40 bar; _ . . 60 bar. 
temperature slope 'when seen from far away' near the vaporising interface which gradually disappears as the supercritical regime is approached. The fast variation of the local pressure $\bar{p}^{0}$ is also apparent in Figure 13 and illustrates the multiscale aspects of the vaporising fronts.

\section{Oxygen/hydrogen flames}

In this section, we investigate the structure of diffusion flames between cold liquid-like oxygen and pure gaseous-like hydrogen. The chemical mechanism of Ó Conaire et al. [61] is used with reaction rates of progress evaluated directly in terms of chemical potentials as given by statistical thermodynamics $[9,36,37]$.

\subsection{Stability limits}

The thermodynamic stability of mixtures is of fundamental importance for the understanding of subcritical as well as supercritical oxygen/hydrogen flame structures. An exhaustive study of the thermodynamic stability of binary mixtures of $\mathrm{O}_{2}$ and $\mathrm{H}_{2} \mathrm{O}$ has been performed using the SRK equation of state in order to understand $\mathrm{O}_{2}$ vaporising interfaces in the presence of $\mathrm{H}_{2} \mathrm{O}$. Numerical simulations indeed show that in $\mathrm{O}_{2} / \mathrm{H}_{2}$ diffusion flames mainly $\mathrm{H}_{2} \mathrm{O}$ is diffusing from the flame front towards the incoming cold oxygen. The other species either disappear in the flame front or are too unstable to survive the cold temperature near the $\mathrm{O}_{2}$ vaporising or pseudo-vaporising interface. There is an exception for the species $\mathrm{H}_{2} \mathrm{O}_{2}$, which is metastable but which is only present in trace amounts near oxygen vaporising interfaces and has not been considered for the benefit of simplicity. Thermodynamic stability domains are obtained by investigating the eigenvalues of the Hessian matrix of entropy $s$ considered as a function of the energy $e$, the specific volume $v$ and the species mass fractions $\mathrm{y}_{1}, \ldots, \mathrm{y}_{n}$.

Liquid/vapour binary phase diagrams for mixtures in the phase plane $\left(T, \bar{p}^{0}\right)$ may be classified into six types according to Van Konynenburg and Scott [62,63]. Following this classification, the liquid/vapour binary phase diagram for $\mathrm{O}_{2}-\mathrm{H}_{2} \mathrm{O}$ is of type III as illustrated in Figure 14. In this diagram, the $\mathrm{O}_{2}$ equilibrium line on the left ends up with the $\mathrm{O}_{2}$ critical point and the $\mathrm{H}_{2} \mathrm{O}$ equilibrium line on the right ends up with the $\mathrm{H}_{2} \mathrm{O}$ critical point. There is also a line of three phase equilibria with a vapour mixture, liquid $\mathrm{O}_{2}$ and liquid $\mathrm{H}_{2} \mathrm{O}$ which almost coincides with the $\mathrm{O}_{2}$ equilibrium line. Then the loci of critical points with a mixture of $\mathrm{O}_{2}$ and $\mathrm{H}_{2} \mathrm{O}$ is divided into two branches. One branch on the right starts from the $\mathrm{H}_{2} \mathrm{O}$ critical point and goes to higher pressure. The other branch on the left side connects the $\mathrm{O}_{2}$ critical point to the end point of the three phase equilibrium line and cannot be seen since these lines nearly coincide for the $\mathrm{O}_{2}-\mathrm{H}_{2} \mathrm{O}$ species pair. Such a Type III phase diagram is associated with the large immiscibility of water and oxygen at low temperature and has been observed between $\mathrm{H}_{2} \mathrm{O}$ and species other than $\mathrm{O}_{2}$, for instance $\mathrm{CO}_{2}, \mathrm{Xe}, \mathrm{H}_{2}$ and $\mathrm{CH}_{4}[64,65]$. On the other hand, there is good miscibility of $\mathrm{H}_{2} \mathrm{O}$ with $\mathrm{NH}_{3}$ and the corresponding phase diagram is then of Type I [64,65]. In Figure 14 are also plotted experimental measurements due to Japas and Franck [64] for water-rich mixture critical points starting from the critical point of $\mathrm{H}_{2} \mathrm{O}$ and good agreement is observed. The corresponding equilibrium diagram in the phase plane $\left(\mathrm{x}_{\mathrm{H}_{2} \mathrm{O}}, \bar{p}^{0}\right)$ is also presented in Figure 15 with again satisfactory agreement with experimental measurements.

We illustrate in Figure 16 the stability phase diagram in the coordinates $\left(v, \mathrm{x}_{\mathrm{H}_{2} \mathrm{O}}\right)$ at the supercritical pressure $\bar{p}^{0}=60$ bar. A study of the entropy Hessian eigenvalues for mixtures of $\mathrm{O}_{2}$ and $\mathrm{H}_{2} \mathrm{O}$ reveals that there are two stable states, a liquid one being almost pure water 


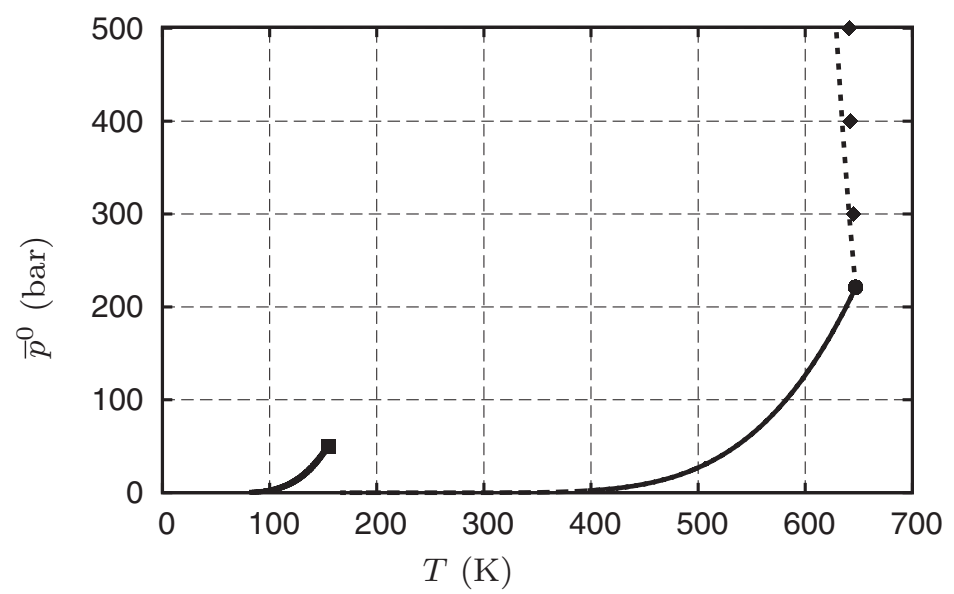

Figure 14. Type III equilibrium phase diagram for $\mathrm{O}_{2} / \mathrm{H}_{2} \mathrm{O}$ : — pure species lines of equilibrium; - $\mathrm{H}_{2} \mathrm{O}$ critical point; $\mathrm{O}_{2}$ critical point; . . - water rich critical points; Experimental measurements.

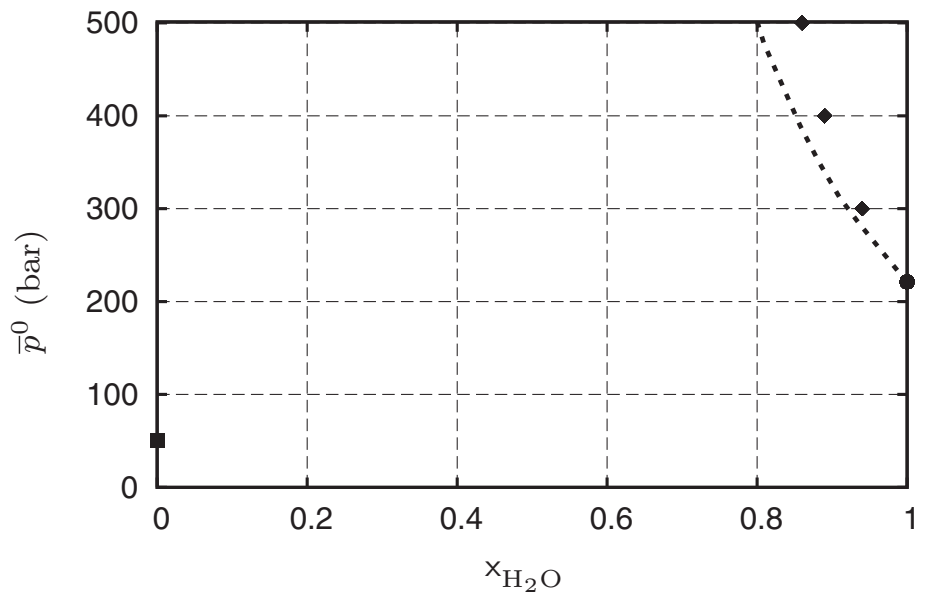

Figure 15. Type III equilibrium phase diagram for $\mathrm{O}_{2} / \mathrm{H}_{2} \mathrm{O}:-\mathrm{H}_{2} \mathrm{O}$ critical point; $\mathrm{O}_{2}$ critical point; . . - water rich critical points; Experimental measurements.

and a fluid one that goes from liquid-like states - where it is nearly pure oxygen - to gaseous-like states where it is a mixture of $\mathrm{O}_{2}$ and $\mathrm{H}_{2} \mathrm{O}$. In Figure 16 are presented the lines of equilibrium between the fluid oxygen containing phase and the liquid-water phase, as well as the chemical stability limit and the mechanical stability limit. The mechanically unstable zone where $\partial_{\rho} \bar{p}<0$ is strictly included in the chemically unstable zone where the matrix $\Lambda$ is not positive definite. The zone between the equilibrium lines and the instability limit corresponds to metastable states and is grey in colour. Note that there is always a single negative eigenvalue of the matrix $\Lambda$ in the unstable zones and thus a single eigenvalue of the entropy Hessian matrix with a positive sign. In other words, there is no new bad eigenvalue when crossing the mechanical stability limit. We also note that for pure water the mechanical and chemical stability zones asymptotically coincide at the top of Figure 16. 


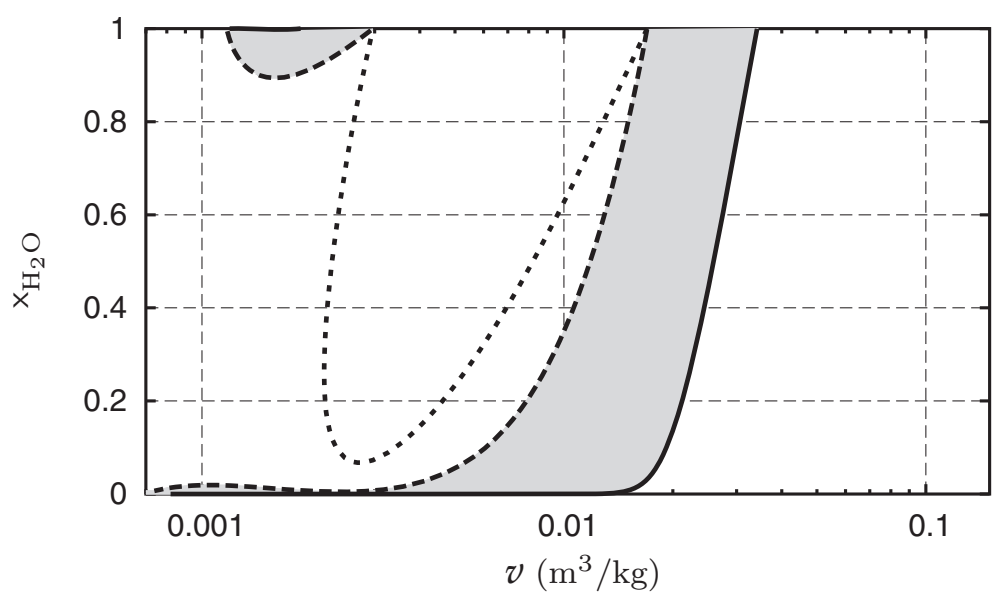

Figure 16. $\mathrm{O}_{2} / \mathrm{H}_{2} \mathrm{O}$ equilibrium diagram at 60 bar: __ lines of equilibrium; _ _ stability limit; _ _ mechanical stability limit. The grey zone corresponds to metastable states.

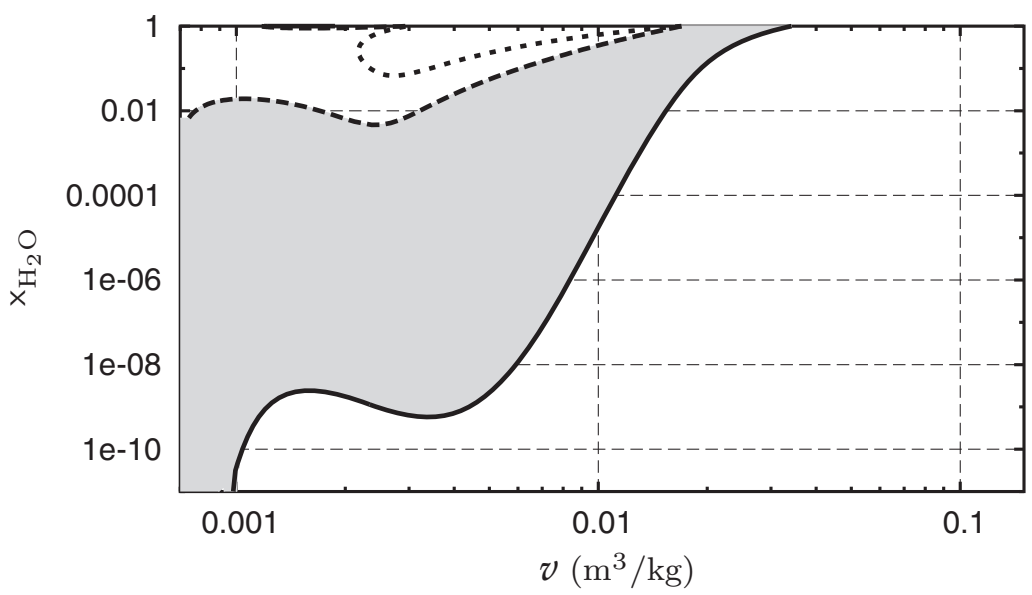

Figure 17. $\mathrm{O}_{2} / \mathrm{H}_{2} \mathrm{O}$ equilibrium diagram with logarithmic coordinates at 60 bar: —u lines of equilibrium; _ _ stability limit; _ . _ mechanical stability limit. The grey zone corresponds to metastable states.

In Figure 16 the unstable zone extends towards small values of the water mole fraction and thus prevents a transition from dense to lighter fluid without entering the forbidden unstable zone if too much water is present. A closer look at the bottom of the diagram of Figure 16 is presented in Figure 17 using logarithmic coordinates and reveals that only 1\% water is sufficient to impose the condensation of water at 60 bar. Numerical experiments indicate that, below 80 bar, there is sufficient water diffusing from the flame core towards the cold zone in order to obtain liquid water. Strictly speaking, the new liquid phase is constituted of water with trace amounts of oxygen and is considered to be pure water for the purpose of simplicity.

A similar stability study is presented in Figures 18 and 19 at the subcritical pressure $\bar{p}^{0}=45$ bar. For such a pressure, three thermodynamically stable phases may be found, namely liquid oxygen, liquid water and a gaseous-like phase that is a mixture of oxygen and 


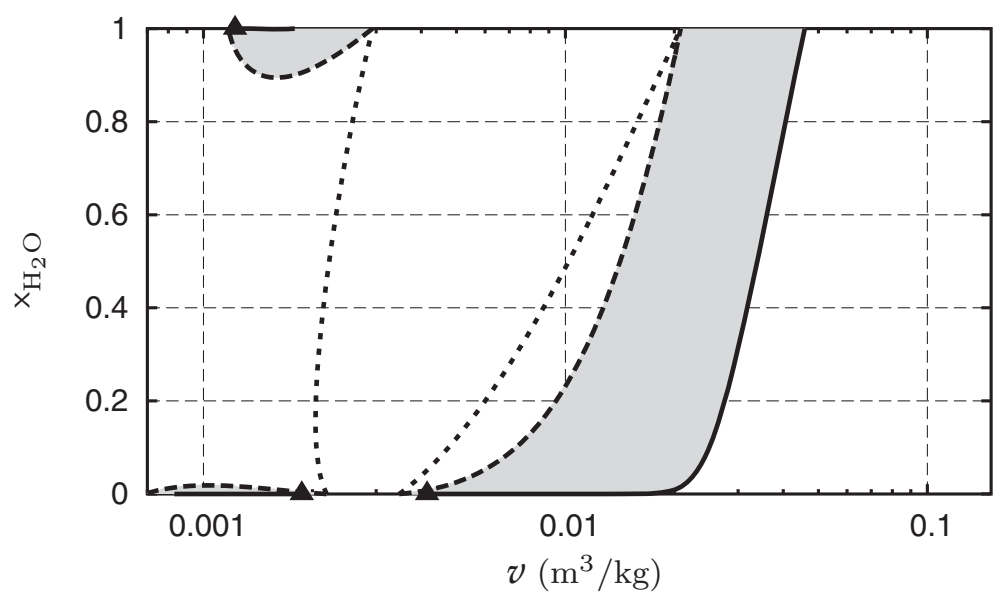

Figure 18. $\mathrm{O}_{2} / \mathrm{H}_{2} \mathrm{O}$ equilibrium diagram at 45 bar: _- lines of equilibrium; _ - stability limit; . . - mechanical stability limit; $\boldsymbol{\Delta}$ three phase equilibrium. The grey zone corresponds to metastable states.

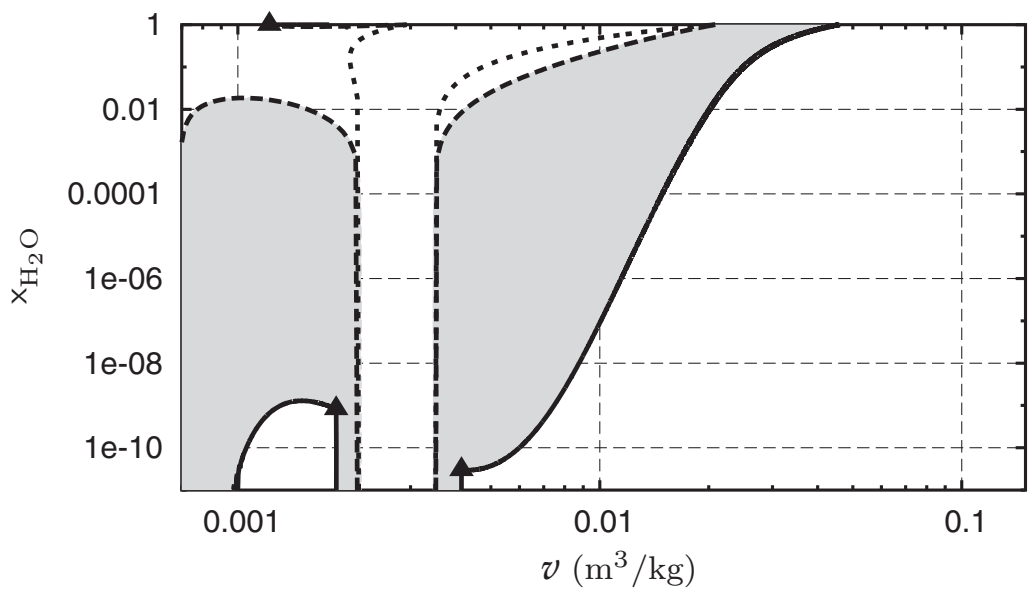

Figure 19. $\mathrm{O}_{2} / \mathrm{H}_{2} \mathrm{O}$ equilibrium diagram with logarithmic coordinates at 45 bar: lines of equilibrium; _- stability limit; _ _ . mechanical stability limit; $\boldsymbol{\Delta}$ three phase equilibrium. The grey zone corresponds to metastable states.

water. Strictly speaking, the liquid phase contains trace amounts of the other species that are not taken into account for the purpose of clarity. There then exist equilibrium lines between each pair of phases as well as a three phase equilibrium point. These equilibrium lines, the three phase equilibrium point, the chemical stability limit and the mechanical stability limit are illustrated in Figures 18 and 19. The zone between the equilibrium lines and the instability limit corresponds to metastable states and is grey in colour. The mechanically unstable zone is again included in the chemically unstable zone and both zones extend from zero up to unity values of the water mole fraction since the pressure is subcritical for both $\mathrm{O}_{2}$ and $\mathrm{H}_{2} \mathrm{O}$. Similar trends are observed as for the supercritical case but now the transition between dense oxygen and lighter oxygen is discontinuous. We also observe again that the 
mechanical and chemical stability limits asymptotically coincide for both pure species $\mathrm{O}_{2}$ and $\mathrm{H}_{2} \mathrm{O}$.

A conclusion of this preliminary analysis is that it is necessary to include a new dispersed phase constituted by liquid-water droplets in the model since it naturally appears below a pressure of about 80 bar. The only species of the new phase to be added is thus $\mathrm{H}_{2} \mathrm{O}(l)$ - assuming that the local pressure remains below $220.6 \mathrm{bar}$ - and the vaporisation/condensation reaction is of the form

$$
\mathrm{H}_{2} \mathrm{O} \rightleftarrows \mathrm{H}_{2} \mathrm{O}(l)
$$

where we have neglected trace amounts of diluted oxygen in $\mathrm{H}_{2} \mathrm{O}(l)$. Such droplets have notably been observed experimentally by Powell using liquid nitrogen [66]. The water condensation mechanism is also complex and involves nucleation of molecular clusters and particle growth through germination [67]. These droplets are only present near the interface and their size has been discussed by Lafon et al. [67]. The droplets are sufficiently small that they follow the fluid local temperature and velocity [67]. As a consequence, a detailed spray simulation including the droplet radius, velocity and temperature [68-70] lay beyond the scope of the present work and only a simplified model is considered. The liquid-water governing equation is of the form

$$
\partial_{t} \rho_{n+1}+\nabla \cdot\left(\rho_{n+1} \boldsymbol{v}\right)=m_{n+1} \omega_{n+1},
$$

where $n+1$ is the $\mathrm{H}_{2} \mathrm{O}(l)$ species index. The source term is modelled according to statistical mechanics [36,37] and approximated in the form

$$
\omega_{n+1} \simeq \mathcal{K}_{I}\left(g_{\mathrm{H}_{2} \mathrm{O}}^{0}-g_{\mathrm{H}_{2} \mathrm{O}(l)}^{0}\right),
$$

since the interface zone is nearly isothermal and with $\mathcal{K}_{I}=\mathcal{K}_{I}^{\prime} \mathrm{y}_{\mathrm{H}_{2} \mathrm{O}(j)}$ where $\mathcal{K}_{I}^{\prime}$ is a constant and $j$ is the gaseous phase when condensation dominates, whereas $j$ is the liquid phase when vaporisation dominates. Such a simplified model of the liquid-water dispersed phase has already been used by Lafon et al. [67] when investigating water condensation around isolated Lox droplets. The new liquid-water phase then mainly acts as source or sink for the species and energy equations [67].

\subsection{Structure of diffusion flames}

In the following, we present typical steady-state diffusion flame structures in the subcritical and supercritical domains. These flames are obtained by flowing pure liquid-like oxygen towards pure gaseous-like hydrogen. We consider a subcritical pressure of $p^{\infty}=45$ bar and a supercritical pressure of $p^{\infty}=60 \mathrm{bar}$ - with respect to an oxygen critical pressure of $p_{c}=50.43$ bar. The strain rate $\alpha=10,000 \mathrm{~s}^{-1}$ corresponds to a velocity decreasing from $500 \mathrm{~cm} \cdot \mathrm{s}^{-1}$ to zero within $0.5 \mathrm{~mm}$, which is representative of strain rates in flames in cryogenic engines. The temperature in the $\mathrm{O}_{2}$ cold stream is $T^{-}=100 \mathrm{~K}$ and in the $\mathrm{H}_{2}$ hot stream $T^{+}=300 \mathrm{~K}$. In all simulations, we have used the reaction mechanism of Ó Conaire et al. [61] and a value of $\kappa=10^{-2} \mathrm{~g}^{-1} \mathrm{~cm}^{7} \mathrm{~s}^{-2}$ of the capillary parameter and $\mathcal{K}_{I}^{\prime}=10^{-2} \mathrm{~mol} \cdot \mathrm{s} \cdot \mathrm{cm}^{-5}$ of the vaporisation/condensation reaction parameter.

Figure 20 presents the temperature $T(\zeta)$ and density $\rho(\zeta)$ as functions of the normal coordinate $\zeta$ in a diffusion flame structure at $p^{\infty}=60$ bar. The density presents a steep variation near the pseudo-vaporising front transitioning from liquid-like to gaseous-like 


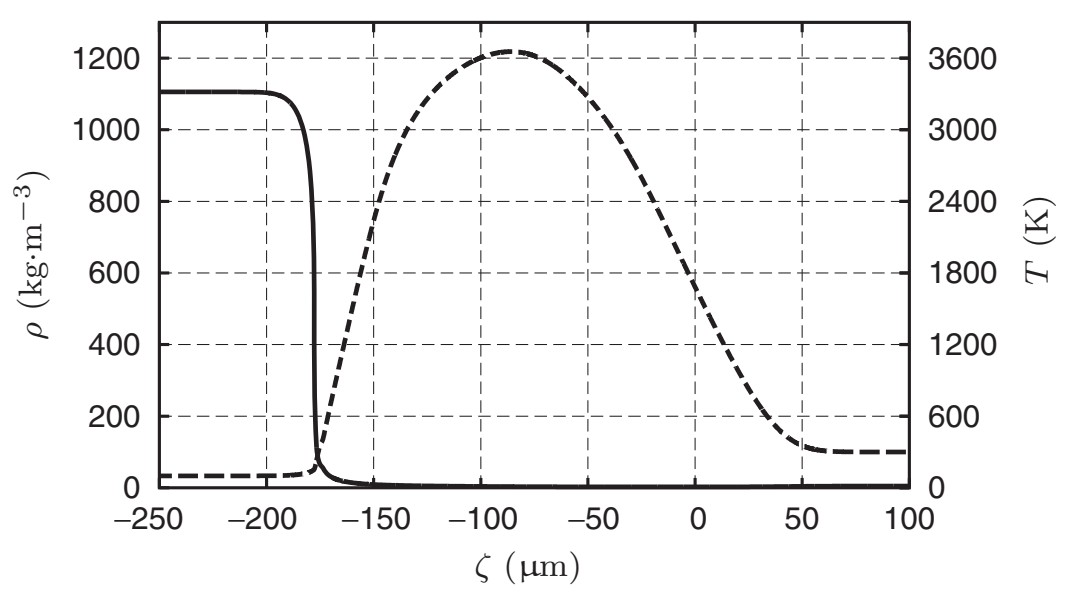

Figure 20. Temperature and density in a diffusion flame at $p^{\infty}=60$ bar: $\_\rho{ }_{-}-T$.

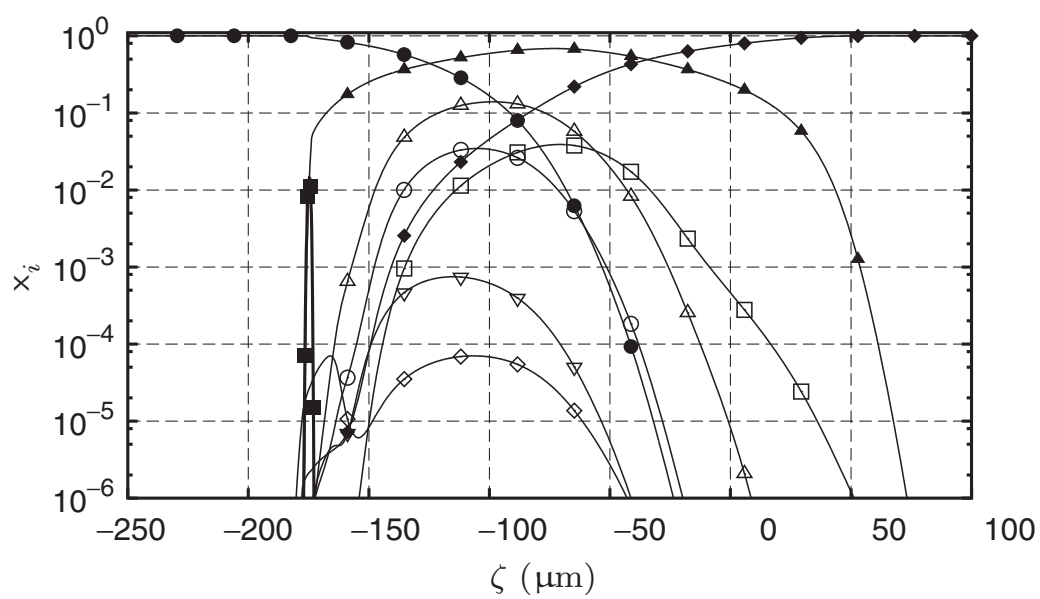

Figure 21. Species mole fractions in a diffusion flame at $p^{\infty}=60$ bar: $\square$ liquid $\mathrm{H}_{2} \mathrm{O} ;$ $\mathrm{H}_{2} \mathrm{O} ; \diamond \mathrm{H}_{2} ; \square \mathrm{H} ; \triangle \mathrm{OH} ; \circ \mathrm{O} ; \nabla \mathrm{HO}_{2} ; \diamond \mathrm{H}_{2} \mathrm{O}_{2}$.

states and the temperature curve is bell-shaped, as is typical of diffusion flames. In Figure 21 are presented the corresponding species mole fractions profiles $\mathbf{x}_{i}(\zeta), i \in \mathfrak{S}$, which are typical of strained diffusion flames but also include liquid water. Since the pressure is $p^{\infty}=$ $60 \mathrm{bar}$, the flame structure is much thinner than at atmospheric pressure. In high pressure flames the reaction $\mathrm{H}+\mathrm{O}_{2}+\mathrm{M} \longrightarrow \mathrm{HO}_{2}+\mathrm{M}$, which decreases the number of moles, dominates $\mathrm{H}+\mathrm{O}_{2} \longrightarrow \mathrm{OH}+\mathrm{O}$ over a larger temperature domain, and this leads to high concentrations of $\mathrm{HO}_{2}$ and $\mathrm{H}_{2} \mathrm{O}_{2}$ radicals [9].

Figure 22 illustrates how the metastable zone is crossed by the flame trajectory in the phase space $\left(v, \mathrm{x}_{\mathrm{H}_{2} \mathrm{O}}\right)$. The pseudo-vaporisation reaction parameters used in the calculations are $\mathcal{K}_{I}^{\prime}=10^{-2} \mathrm{~mol} \cdot \mathrm{s} \cdot \mathrm{cm}^{-5}, \mathcal{K}_{I}^{\prime}=10^{-4} \mathrm{~mol} \cdot \mathrm{s} \cdot \mathrm{cm}^{-5}, \mathcal{K}_{I}^{\prime}=10^{-6} \mathrm{~mol} \cdot \mathrm{s} \cdot \mathrm{cm}^{-5}$ and $\mathcal{K}_{I}^{\prime}=10^{-7} \mathrm{~mol} \cdot \mathrm{s} \cdot \mathrm{cm}^{-5}$. For the lower value $\mathcal{K}_{I}^{\prime}=10^{-8} \mathrm{~mol} \cdot \mathrm{s} \cdot \mathrm{cm}^{-5}$, the flame trajectory would enter the forbidden unstable zone. In other words, there is no hope of avoiding the presence of liquid water droplets at such a pressure. 


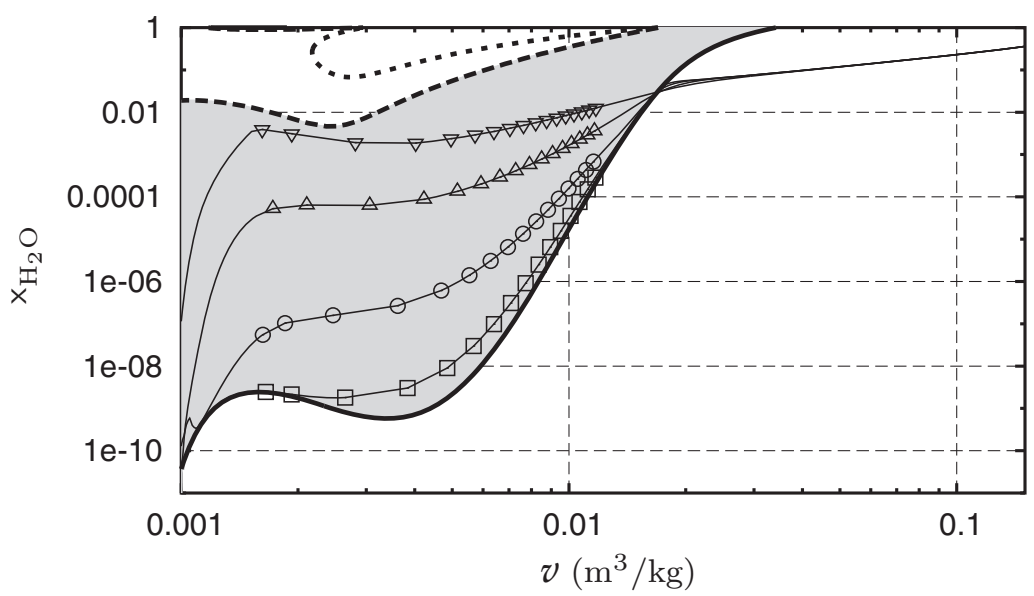

Figure 22. Phase space diagram and flame trajectory at 60 bar: __ lines of equilibrium; _ - stability limit; - . - mechanical stability limit; $\square \mathcal{K}_{I}^{\prime}=10^{-2} ; \circ \mathcal{K}_{I}^{\prime}=10^{-4} ; \Delta \mathcal{K}_{I}^{\prime}=10^{-6} ; \nabla \mathcal{K}_{I}^{\prime}=10^{-7}$. The grey zone corresponds to metastable states.

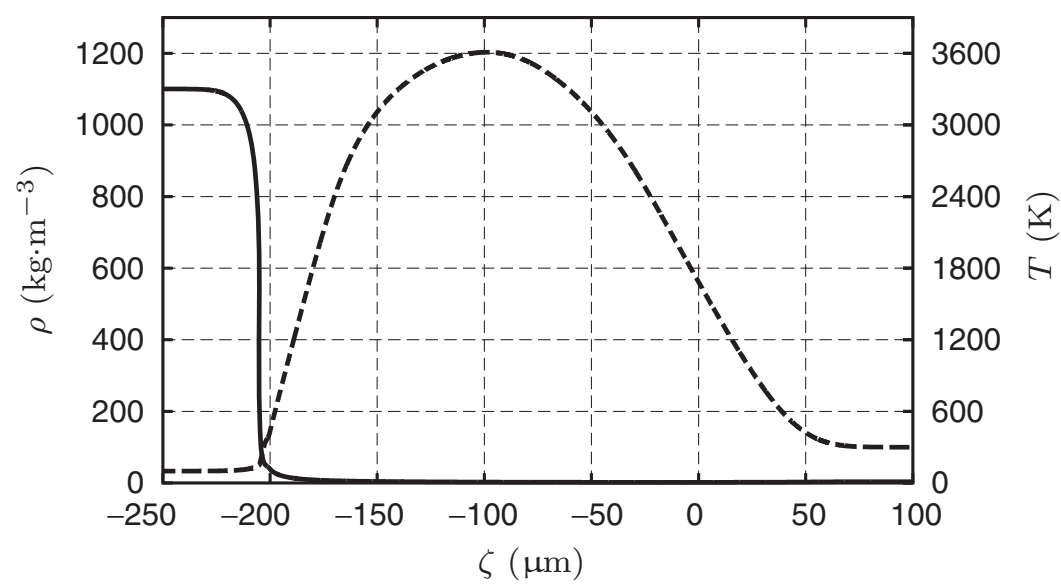

Figure 23. Temperature and density in a diffusion flame at $p^{\infty}=45$ bar: $\_\rho{ }_{-}-T$.

We present in Figure 23 the temperature $T(\zeta)$ and density $\rho(\zeta)$ as functions of $\zeta$ in a diffusion flame structure at $p^{\infty}=45$ bar. The density again presents a steep variation at the vaporising front transitioning from liquid to gaseous states. Figure 24 shows the species mole fractions profiles $\mathrm{x}_{i}(\zeta), i \in \mathfrak{S}$, as functions of the flame normal coordinate $\zeta$. The corresponding profiles are similar to those observed at the higher ambient pressure $p^{\infty}=60$ bar with a sharp variation of density in the vaporising zone, the appearance and disappearance of liquid water, the diffusion flame type bell shaped temperature distribution, and analogous species mole fraction profiles typical of oxygen/hydrogen diffusion flames. There is also a smooth transition between the subcritical and supercritical conditions in the calculations thanks to the diffuse interface model.

The flame structure around the vaporising interface is presented on Figure 25. We notably observe the condensation of water coming from the flame front by diffusion that cannot enter further into liquid oxygen since these fluids are largely immiscible at low 


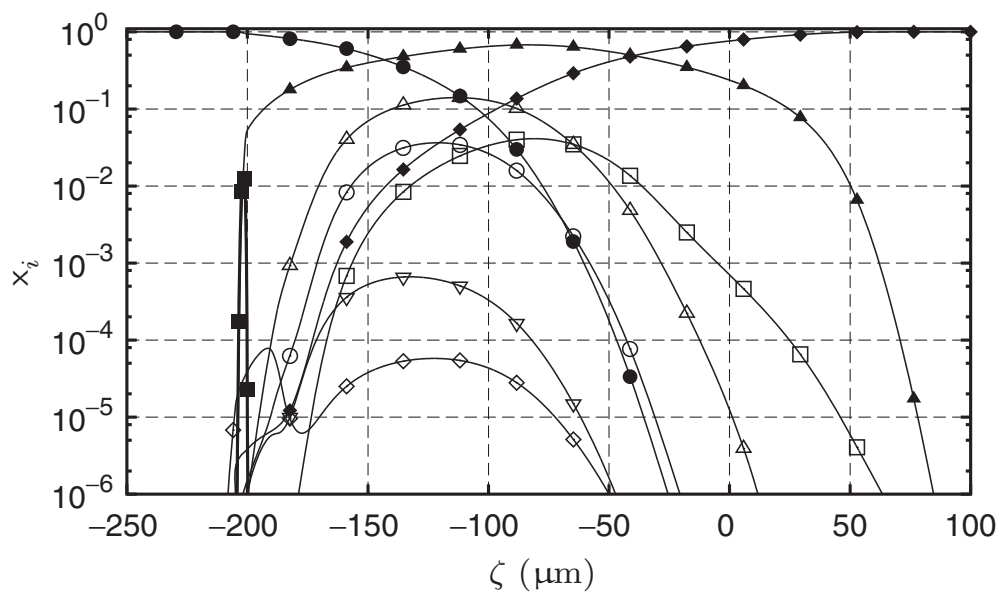

Figure 24. Species mole fractions in a diffusion flame at $p^{\infty}=45$ bar: $\boldsymbol{H}_{2} \mathrm{O}(l) ; \bullet \mathrm{O}_{2} ; \boldsymbol{\Delta} \mathrm{H}_{2} \mathrm{O}$; $\mathrm{H}_{2} ; \square \mathrm{H} ; \triangle \mathrm{OH} ; \circ \mathrm{O} ; \nabla \mathrm{HO}_{2} ; \diamond \mathrm{H}_{2} \mathrm{O}_{2}$.

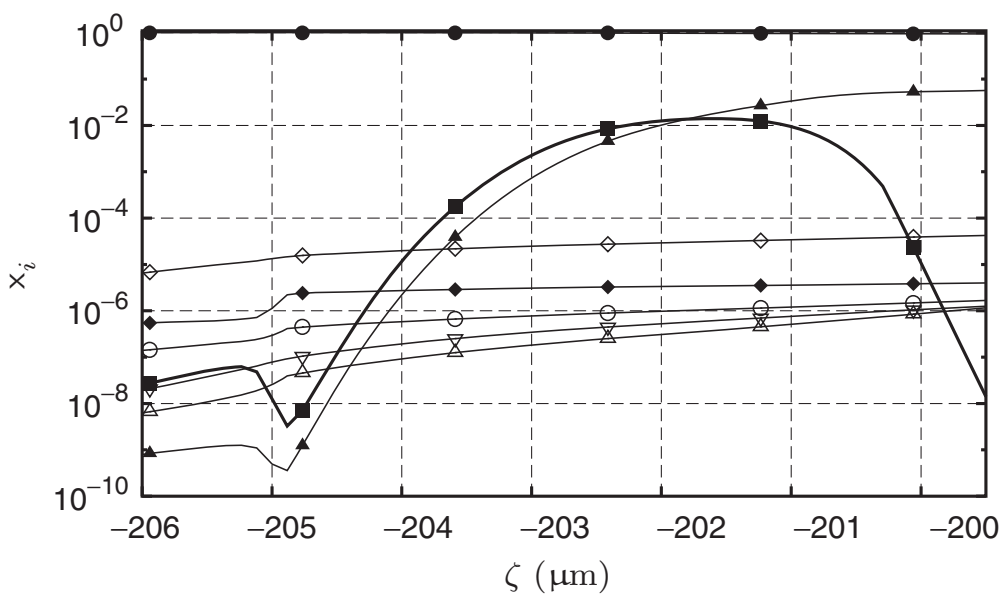

Figure 25. Species mole fractions near the vaporising interface in a diffusion flame at $p^{\infty}=45$ bar: $\mathrm{H}_{2} \mathrm{O}(l) ; \bigcirc \mathrm{O}_{2} ; \Delta \mathrm{H}_{2} \mathrm{O} ; \diamond \mathrm{H}_{2} ; \triangle \mathrm{OH} ; \circ \mathrm{O} ; \nabla \mathrm{HO}_{2} ; \diamond \mathrm{H}_{2} \mathrm{O}_{2}$

temperature. The chemical instability associated with water at low temperature has been taken into account by including liquid water as a dispersed phase but it still remains a thermodynamic instability mainly associated with the phase transition of oxygen. If oxygen were pure the instability would be a mechanical instability, but in the presence of other species it is generally a chemical instability as indicated by the phase diagrams. The continuous transition between liquid oxygen and gaseous oxygen is here due to the second gradient model of van der Waals type which removes the singularity associated with the wrong sign eigenvalue in the entropy Hessian matrix [71].

Figure 26 illustrates how the unstable zone is crossed by the flame trajectory with the help of the second gradient stabilizing terms. The various flame trajectories in the phase space $\left(v, \mathrm{x}_{\mathrm{H}_{2} \mathrm{O}}\right)$ correspond to the pseudo-vaporisation reaction parameters $\mathcal{K}_{I}^{\prime}=$ $10^{-2}, \mathcal{K}_{I}^{\prime}=10^{-4}$ and $\mathcal{K}_{I}^{\prime}=10^{-6}$. In comparison with the supercritical situation where the 


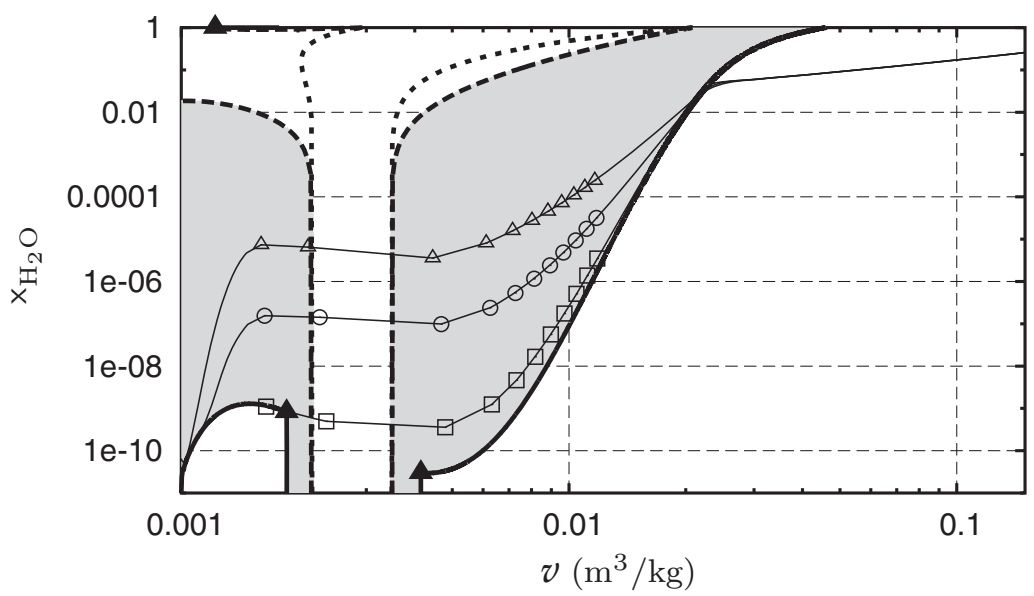

Figure 26. Phase space diagram and flame trajectory at $p^{\infty}=45 \mathrm{bar} ; \quad$ lines of equilibrium; _stability limit; . . . mechanical stability limit; $\boldsymbol{\Delta}$ three phase equilibrium; $\square \mathcal{K}_{I}^{\prime}=10^{-2} ; \circ \mathcal{K}_{I}^{\prime}=10^{-4}$; $\Delta \mathcal{K}_{I}^{\prime}=10^{-6}$. The grey zone corresponds to metastable states.

metastable zone was crossed and the forbidden zone avoided with the help of the presence of liquid water, we observe here that the upper part of the forbidden unstable zone is still avoided with the help of liquid water, whereas the lower part of the forbidden zone is crossed with the help of the stabilizing properties of the second gradient terms [71].

\section{Conclusion}

We have introduced a diffuse-interface all-pressure flame model that transitions smoothly from subcritical to supercritical conditions. This model involves a non-equilibrium diffuse interface embeded into a non-ideal reactive fluid with multicomponent fluxes written in thermodynamic form as well as liquid water as a dispersed phase. The model has been simplified using a small Mach number limit with self-similar assumptions typical of strained flows.

Numerical simulations of oxygen interfaces, oxygen/hydrogen diffusion flames, as well as stability diagrams have confirmed that the resulting model transitions smoothly from subcritical to supercritical conditions. We have further established that the SRK equation of state is able to reproduce the phase stability diagram of $\mathrm{O}_{2} / \mathrm{H}_{2} \mathrm{O}$ mixtures, which is of type III according to the Van Konynenburg and Scott classification.

A first extension of the present work of high scientific interest would be to focus on detailed simulations of interfaces including accurate capillary coefficients, nanolength grid resolution, and/or a detailed description of the liquid-water dispersed phase with comparisons with experiment.

Another perspective of high scientific interest would be to perform direct numerical simulations as well as large eddy simulations of the transition between subcritical and supercritical flames using the compressible formulation, which is fully valid in several dimensions.

\section{Disclosure statement}

No potential conflict of interest was reported by the authors. 


\section{References}

[1] M. Habiballah, M. Orain, F. Grisch, L. Vingert, and P. Gicquel, Experimental studies of high pressure cryogenic flames on the MASCOTTE facility, Combust. Sci. Technol. 178 (2006), pp. 101-128.

[2] S. Candel, M. Juniper, G. Singla, P. Scouflaire, and C. Rolon, Structure and dynamics of cryogenic flames at supercritical pressure, Combust. Sci. Technol. 178 (2006), pp. 161-192.

[3] A.M. Saur, F. Behrendt, and E.U. Franck, Calculation of high pressure counterflow diffusion flame up to 3000 bar, Ber. Bunsen. physik. Chem. 97 (1993), pp. 900-908.

[4] J. Daou, P. Haldenwang, and C. Nicoli, Supercritical burning of liquid oxygen (LOX) droplets with detailed chemistry, Combust. Flame 101 (1995), pp. 153-169.

[5] M. El Gamal, E. Gutheil, and J. Warnatz, The structure of laminar premixed $\mathrm{H}_{2}$-air flames at elevated pressures, Z. Phys. Chem. 214 (2000), pp. 419-435.

[6] N.A. Okongo and J. Bellan, Direct numerical simulation of a transitional supercritical binary mixing layer: Heptane and nitrogen, J. Fluid Mech. 464 (2002), pp. 1-34.

[7] G. Ribert, N. Zong, V. Yang, L. Pons, N. Darabiha, and S. Candel, Counterflow diffusion flames of general fluids: Oxygen/hydrogen mixtures, Combust. Flame 154 (2008), pp. 319-330.

[8] L. Pons, N. Darabiha, S. Candel, G. Ribert, and V. Yang, Mass transfer and combustion in transcritical non-premixed counterflows, Combust. Theory Model. 13 (2009), pp. 57-81.

[9] V. Giovangigli, L. Matuszewski, and F. Dupoirieux, Detailed Modeling of Transcritical Planar $\mathrm{H}_{2}-\mathrm{O}_{2}-\mathrm{N}_{2}$ flames, Combust. Theory Model. 15 (2011), pp. 141-182.

[10] V. Giovangigli and L. Matuszewski, Numerical simulation of transcritical strained laminar flames, Combust. Flame 159 (2012), pp. 2829-2840.

[11] J. Bellan, Theory, modeling and analysis of turbulent supercritical mixing, Combust. Sci. Technol. 178 (2006), pp. 253-281.

[12] J.C. Oefelein, Thermophysical characteristics of shear-coaxial $\mathrm{LOX}-\mathrm{H}_{2}$ flames at supercritical pressure, Proc. Combust. Inst. 30 (2005), pp. 2929-2937.

[13] T. Schmitt, Y. Méry, M. Boileau, and S. Candel, Large-eddy simulation of oxygen/methane flames under transcritical conditions, Proc. Combust. Inst. 33 (2011), pp. 1383-1390.

[14] J.D. van der Waals, Thermodynamische Theorie der Capillariteit in de Onderstelling van Continue Dichtheidsverandering, Verhandlingen der Koninglijke Akademie van Weten-schappen, Amsterdam, 1893 [In Dutch.] English translation by J.S. Rowlinson: The thermodynamic theory of capillarity under the hypothesis of a continuous variation of density, J. Statist. Phys., 20 (1979), pp. 197-244.

[15] J.D. van der Waals, Thermodynamisch Theorie der Kapillariät unter Voraussetzung stetiger Dichteanderung, Z. Phys. Chem. 13 (1894), pp. 657-725.

[16] D.J. Korteweg, Sur la forme que prennent les équations du mouvement fluide si l'on tient compte de forces capillaires causées par les variations de densité considérables mais continues et sur la théorie de la capillarité dans l'hypothèse d'une variation continue de la densité, Arch. Neerl. Sci. Exactes 6 (1901), pp. 1-20.

[17] J.W. Cahn and J.E. Hilliard, Free energy of a nonuniform system. I. Interfacial free energy, J. Chem. Phys. 28 (1958), pp. 258-267. Available at http://dx.doi.org/10.1063/1.1744102.

[18] J.W. Cahn and J.E. Hilliard, Free energy of a nonuniform system. II. Thermodynamic basis, J. Chem. Phys. 30 (1959), pp. 1121-1124. Available at http://dx.doi.org/10.1063/1.1730145.

[19] J.S. Rowlinson and B. Widom, Molecular Theory of Capillarity, Dover, Mineola, TX, 2002.

[20] P. Seppecher, Moving contact line in the Cahn-Hilliard theory, Int. J. Eng. Sci. 34 (1996), pp. 977-992. Available at http://dx.doi.org/10.1016/0020-7225(95)00141-7.

[21] D.M. Anderson, G.B. McFadden, and A.A. Wheeler, Diffuse interface methods in fluid mechanics, Annu. Rev. Fluid Mech. 30 (1998), pp. 139-165.

[22] D. Jamet, O. Lebaigue, N. Coutris, and J.M. Delhaye, The second gradient method for the direct numerical simulation of liquid-vapor flows with phase change, J. Comput. Phys. 169 (2001), pp. 624-651.

[23] D. Jamet, Diffuse interface models in fluid mechanics (2005). Available at https://www. researchgate.net/profile/Didier_Jamet/publication/254424182_Diffuse_interface_models_in_ uid_mechanics/links/53f327960cf256ab87b07ada.pdf.

[24] H. Lin, Y.-Y. Duan, and Q. Min, Gradient theory modeling of surface tension for pure fluids and binary mixtures, Fluid Phase Equilib. 254 (2007), pp. 75-90. Available at http://dx.doi.org/10.1016/j.fluid.2007.02.013. 
[25] R.N. Dahms and J.C. Oefelein, On the transition between two-phase interface dynamics in multicomponent fluids at supercritical pressures, Phys. Fluids 25 (2013), pp. 1-24.

[26] J.C. Oefelein, R.N. Dahms, G. Lacaze, J.L. Manin, and L.M. Pickett, Effects of pressure on the fundamental physics of fuel injection in diesel engines, in Proceedings of the 12th International Conference on Liquid Atomization and Spray Systems (ICLASS 2012), 2-6 September 2012, Heidelberg.

[27] R.I. Nigmatulin, Methods of mechanics of a continuous medium for the description of multiphase mixtures, J. Appl. Math. Mech. 34 (1970), pp. 1197-1112.

[28] M.R. Baer and J.W. Nunziato, A two-phase mixture theory for the deflagration-to-detonation transition (DDT) in reactive granular media, Int. J. Multiphase Flow 12 (1986), pp. 861-889.

[29] D.A. Drew and S.L. Passman, Theory of Multicomponent Fluids, Applied Mathematical Sciences 135, Springer-Verlag, New York, 1999.

[30] A.K. Kapila, R. Menikoff, J.B. Bdzil, S.F. Son, and D.S. Stewart, Two-phase modeling of the deflagration-to-detonation transition in reactive granular materials: Reduced equations, Phys. Fluids 13 (2001), pp. 3002-3024.

[31] G. Allaire, S. Clerc, and S. Kokh, A five equation models for the simulation of interfaces between compressible fluids, J. Comput. Phys. 181 (2002), pp. 577-616.

[32] R. Saurel, F. Petitpas, and R. Abgrall, Modeling phase transition in metastable liquids, application to cavitating and flashing flows, J. Fluid Mech. 607 (2008), pp. 313-350.

[33] R. Saurel, F. Petitpas, and R. A. Berry, Simple and efficient relaxation methods for interfaces separating compressible fluids, cavitating flows and shock in multiphase mixtures, J. Comput. Phys. 228 (2009), pp. 1678-1712.

[34] A. Ambroso, C. Chalons, and P.-A. Raviart, A Godunov type method for the seven-equation model of compressible two-phase flow, Computers \& Fluids 54 (2012), pp. 67-91.

[35] S.R. de Groot and P. Mazur, Non-Equilibrium Thermodynamics, Dover, Mineola, TX, 1984.

[36] M.R. Marcelin, Sur la mécanique des phénomènes irréversibles, C.R. Acad. Sci. Paris (December 1910), pp. 1052-1055.

[37] J. Keizer, Statistical Thermodynamics of Nonequilibrium Processes, Springer-Verlag, New York, 1987.

[38] V. Giovangigli, Multicomponent flow modeling, Birkhaüser, Boston, MA, 1999.

[39] O. Redlich and J.N.S. Kwong, On the thermodynamics of solutions. V. An equation of state. Fugacities of gaseous solutions, Chem. Reviews 44 (1949), pp. 233-244.

[40] G.S. Soave, Equilibrium constants from a modified Redlich-Kwong equation of state, Chem. Eng. Sci. 27 (1972), pp. 157-172.

[41] V. Giovangigli and L. Matuszewski, Supercritical fluid thermodynamics from equations of state, Physica D 241 (2012), pp. 649-670.

[42] A. Congiunti, C. Bruno, and E. Giacomazzi, Supercritical combustion properties, in 11th Aerospace Sciences Meeting and Exhibit, 6-9 January 2003, Reno, NV, AIAA-2003-478.

[43] P. Colonna and P. Silva, Dense gas thermodynamic properties of single and multicomponent fluids for fluid dynamics simulations, J. Fluid Eng. 125 (2003), pp. 414-427.

[44] W.A. Cañas-Marín, U.E. Guerrero-Aconcha, and J.D. Ortiz-Arango, Comparison of different cubic equations of state and combination rules for predicting residual chemical potential of binary and ternary Lennard-Jones mixtures, Fluid Phase Equilib. 234 (2005), pp. 42-50.

[45] W.A. Cañas-Marín, J.D. Ortiz-Arango, U.E. Guerrero-Aconcha, and C.P. Soto-Tavera, Thermodynamic derivative properties and densities for hyperbaric gas condensates: SRK equation of state predictions versus Monte Carlo data, Fluid Phase Equilib. 253 (2007), pp. 147-154.

[46] Giovangigli and L. Matuszewski, Mathematical modeling of supercritical multicomponent reactive fluids, Math. Mod. Meth. App. Sci. 23 (2013), pp. 2193-2251.

[47] V. Giovangigli, Convergent iterative methods for multicomponent diffusion, IMPACT Comput. Sci. Engng. 3 (1991), pp. 244-276.

[48] V. Giovangigli, Mass conservation and singular multicomponent diffusion algorithms, IMPACT Comput. Sci. Engng. 2 (1990), pp. 73-97.

[49] A. Ern and V. Giovangigli, Multicomponent transport algorithms, Lecture Notes in Physics, Monograph m24, Springer-Verlag, Heidelberg, 1994.

[50] A. Ern and V. Giovangigli, Projected iterative algorithms with application to multicomponent transport, Linear Algebra Appl. 250 (1997), pp. 289-315.

[51] A. Ern and V. Giovangigli, Thermal diffusion effects in hydrogen-air and methane-air flames, Combust. Theory Model. 2 (1998), pp. 349-372. 
[52] H. Van Beijeren and M. H. Ernst, The modified Enskog equations for mixtures, Phys. A 70 (1973), pp. 225-242.

[53] V.I. Kurochkin, S.F. Makarenko, and G.A. Tirskii, Transport coefficients and the Onsager relations in the kinetic theory of dense gas mixtures, J. Appl. Mech. Tech. Phys. 25 (1984), pp. 218-225.

[54] J.F. Ely and H.J. Hanley, Prediction of transport properties. 2. Thermal conductivity of pure fluids and mixtures, Indus. Eng. Chem. Fund. 22 (1983), pp. 90-97.

[55] T.H. Chung, M. Ajlan, L.L. Lee, and K.E. Starling, Generalized multiparameter correlation for nonpolar and polar fluid transport properties, Ind. Eng. Chem. Res. 27 (1988), pp. 671-679.

[56] L.P. Kadanoff and J. Swift, Transport coefficients near the liquid-gas critical point, Phys. Rev. 166 (1968), pp. 89-101.

[57] J.V. Sengers, Transport processes near the critical point of gases and binary liquids in the hydrodynamic regime, Ber. Bunsen. physik. Chem. 76 (1972), pp. 234-249.

[58] M. D. Smooke, Solution of burner stabilized premixed laminar flames by boundary value methods, J. Comp. Phys. 48 (1982), pp. 72-105.

[59] M.D. Smooke, The computation of laminar flames, Proc. Combust. Inst. 34 (2013), pp. 65-98.

[60] V. Giovangigli and M. D. Smooke, Adaptive continuation algorithms with application to combustion problems, Appl. Numer. Math. 5 (1989), pp. 305-331.

[61] M. Ó Conaire, H.J. Curran, J.M. Simmie, W.J. Pitz, and C.K. Westbrook, A comprehensive modeling study of hydrogen oxidation, Int. J. Chem. Kin. 36 (2004), pp. 603-622.

[62] P.H. Van Konynenburg and R.L. Scott, Critical lines and phase equilibria in binary van der Waals mixtures, Phil. Trans. Roy. Soc. London Ser. A 298 (1980) pp. 495-540.

[63] J.M. Prausnitz, R.N. Lichtenthaler, and E.G. de Avezo, Molecular thermodynamics of fluidphase equilibria, Prentice-Hall, Upper Saddle River, NJ, 1999.

[64] M.L. Japas and E.U. Franck, High pressure phase equilibria and PVT-data of the water-oxygen system including water-air to $673 \mathrm{~K}$ and $250 \mathrm{MPa}$, Ber. Bunsen. physik. Chem. 89 (1985) pp. 1268-1275. Available at http://dx.doi.org/10.1002/bbpc.19850891206

[65] H. Weingärtner and E.U. Franck, Supercritical water as a solvent, Angew. Chem. Int. Ed. 44 (2005) pp. 2672-2692.

[66] E.E. Powell, Simulation of water vapor condensation on LOX droplet surface using liquid nitrogen, NASA/ASEE Summer Faculty Fellowship Program, N89-21726, XXIII, 1988.

[67] P. Lafon, H. Meng, V. Yang, and M. Habiballah, Vaporization of liquid oxygen (LOX) droplets in hydrogen and water environments under sub- and super-critical conditions, Combust. Sci. Technol. 180 (2008), pp. 1-26.

[68] S.C. Li, P.A. Libby, and F.A. Williams, Spray structure in counterflowing streams with and without a flame, Combust. Flame 94 (1993), pp. 161-177.

[69] M. Massot, M. Kumar, M.D. Smooke, and A. Gomez, Spray counterflow diffusion flames of heptane: Experiments and computation with detailed kinetics and transport, Proc. Combust. Inst. 27 (1998), pp. 1975-1983.

[70] R. Bendahklia, V. Giovangigli, and D. Rosner, Soret effects in laminar counterflow spray diffusion flames, Combust. Theory Model. 6 (2002), pp. 1-17.

[71] S. Benzoni-Gavage, R. Danchin, S. Descombes, and D. Jamet, Structure of Korteweg models and stability of diffuse interfaces, Interfaces \& Free Bound. 7 (2005), pp. 371-414.

\section{Appendix A. Equilibrium interfaces}

We discuss in this section the equations governing equilibrium interfaces and the link with the non-equilibrium model presented in Sections 2 and 3.

We first consider the situation of a single species or equivalently the simplified formulation of Section 2.4. In order to obtain the equilibrium structure of the interface, the entropy has to be maximised for a given amount of mass and energy. (Equivalently, we may minimise the free energy for a given amount of mass at constant temperature.) Using the method of Lagrange multipliers, we write that the infinitesimal variation of interfacial entropy vanishes [23]

$$
\delta \int\left(\mathcal{S}+\alpha_{e} \mathcal{E}+\alpha_{\rho} \rho\right) \mathrm{d} \mathbf{x}=0
$$


where $\delta$ is the infinitesimal variation symbol and $\alpha_{\rho}, \alpha_{e}$ are Lagrange multipliers. Using $\mathcal{E}=$ $\mathcal{F}+T \mathcal{S}=\mathcal{F}+T \mathcal{S}^{0}$, the differential expression (2), and a few integrations by parts, we obtain that

$$
\int\left(\left(1+T \alpha_{e}\right) \delta \mathcal{S}^{0}+\left(\alpha_{e}\left(g^{0}-\nabla \cdot \boldsymbol{\phi}\right)+\alpha_{\rho}\right) \delta \rho\right) \mathrm{d} \mathbf{x}=0 .
$$

Since the variations $\delta \mathcal{S}^{0}$ and $\delta \rho$ are arbitrary, we deduce the Euler-Lagrange equations $\left(1+T \alpha_{e}\right)=0$ and $\alpha_{e}\left(g^{0}-\nabla \cdot(\kappa \nabla \rho)\right)+\alpha_{\rho}=0$ since $\phi=\kappa \nabla \rho$. The first implies that the interface is isothermal with $T=-1 / \alpha_{e}$. Further considering now a planar interface for the sake of simplicity, denoting by ' the derivation with respect to the normal coordinate, and assuming that $\kappa$ is a constant, we obtain that

$$
g^{0}-\kappa \rho^{\prime \prime}=\text { Const. }=g^{\infty},
$$

where $g^{\infty}$ denotes the common value of $g^{0}$ in both fluids at $-\infty$ and $+\infty$. Multiplying this relation by $\rho^{\prime}$, integrating through the interface, making use of $\partial_{\rho} \mathcal{F}^{0}=g^{0}$, we next obtain that

$$
\mathcal{F}^{0}-\frac{1}{2} \kappa \rho^{\prime 2}-g^{\infty} \rho=\text { Const. }
$$

It has been assumed here that the density $\rho$ goes towards the liquid and gas density values at both ends so that the spatial derivative $\rho^{\prime}=\partial_{\zeta} \rho$ vanishes at both ends. Combining these relations, we obtain that

$$
p^{0}+\frac{1}{2} \kappa \rho^{\prime 2}-\kappa \rho \rho^{\prime \prime}=p^{\infty}
$$

where $p^{\infty}$ denotes the common value of pressure for both fluids, which coincides for a one-dimensional interface with the momentum conservation equation when it is assumed that $v=0$ and $T=$ Const.

Conversely, let us consider a steady interface with $\boldsymbol{v}=0, T=$ Const., with a one-dimensional structure. Using the momentum conservation equation we then obtain that (A4) holds. Deriving with respect to the interface normal coordinate, we obtain that

$$
\left(\partial_{\rho} p^{0}\right)_{T} \rho^{\prime}-\kappa \rho \rho^{\prime \prime \prime}=0 .
$$

Using then the thermodynamic relation $\left(\partial_{\rho} p^{0}\right)_{T}=\rho\left(\partial_{\rho} g^{0}\right)_{T}$, simplifying by $\rho$, we may integrate with respect to the interface normal coordinate to recover (A2) and a further integration - previously multiplying by $\rho^{\prime}$ - then yields (A3). Therefore the interface model considered in this work coincides with an equilibrium model provided it is steady, without convection, isothermal and with constant limits at both interface ends, i.e. without gradients at the boundaries. One may observe, however, that the presence of convective terms is not a significant limitation because the Mach numbers usually involved are rather small and similarly the simulations of Sections 4 and 5.2 further show that temperature variations are modest in the vaporising or pseudo-vaporising zones. The assumption that is not always satisfied is that the gradients at the interface boundaries are vanishing.

Similar conclusions may be reached for a multicomponent interface. Proceeding in an analogous way, we have to to maximise the interfacial entropy for a given energy and given masses for each chemical species. Proceeding similarly, we have the extremal property

$$
\delta \int\left(\mathcal{S}+\alpha_{e} \mathcal{E}+\sum_{k \in \mathfrak{S}} \alpha_{k} \rho_{k}\right) \mathrm{d} \mathbf{x}=0
$$


where $\alpha_{k}, k \in \mathfrak{S}$, and $\alpha_{e}$ are Lagrange multipliers. Using then $\mathcal{E}=\mathcal{F}+T \mathcal{S}^{0}$, the differential expression (2), and a few integrations by parts, we obtain that

$$
\int_{\mathcal{V}}\left(\left(1+T \alpha_{e}\right) \delta \mathcal{S}^{0}+\sum_{k \in \mathfrak{S}}\left(\alpha_{e}\left(g_{k}^{0}-\nabla \cdot \boldsymbol{\phi}_{k}\right)+\alpha_{k}\right) \delta \rho_{k}\right) \mathrm{dx}=0 .
$$

Since the variations $\delta \mathcal{S}^{0}$ and $\delta \rho_{k}, k \in \mathfrak{S}$, are arbitrary we obtain that $\left(1+T \alpha_{e}\right)=0$ and $\alpha_{e}\left(g_{k}^{0}-\nabla\right.$. $\left.\boldsymbol{\phi}_{k}\right)+\alpha_{k}=0, k \in \mathfrak{S}$. We have thus recovered that the interface is isothermal and that the generalised chemical potentials $\widetilde{g}_{k}=g_{k}^{0}-\nabla \cdot \boldsymbol{\phi}_{k}$ are constants through the interface. It is then found that the interface model of Section 2.1 coincides with the model obtained at equilibrium provided the interface is steady, without convection, isothermal, without gradients of chemical potentials, without chemistry and with constant limits at both interface limits. We again observe that convection is not a significant limitation because of small Mach numbers and the simulations of Sections 4 and 5.2 also suggest that temperature variations are modest in the interfaces. The assumptions that are not always satisfied is that the gradients at the interface boundaries are vanishing as well as the absence of chemistry.

We finally note that upon writing the partial densities in the form $\rho_{k}=\rho \mathbf{y}_{k}$, assuming that the species mass fractions are essentially invariants in the interface, so that $\rho_{k}^{\prime} \simeq \mathrm{y}_{k} \rho^{\prime}$, and summing the relations $g_{k}^{0}-\boldsymbol{\phi}_{k}^{\prime}=$ Cte we recover that $g^{0}-\bar{\kappa} \rho^{\prime \prime} \simeq$ Cte, where $\bar{\kappa}$ is an average value of the species capillary parameters. The simplified model will thus be a relevant model provided that the mass fractions are essentially unchanged in the interface. 